\title{
Bulk Viscous Cosmological Model in Brans-Dicke Theory with New Form of Time Varying Deceleration Parameter
}

\author{
G. P. Singh and Binaya K. Bishi \\ Department of Mathematics, Visvesvaraya National Institute of Technology, Nagpur 440010, India \\ Correspondence should be addressed to Binaya K. Bishi; binaybc@gmail.com
}

Received 25 March 2017; Accepted 22 May 2017; Published 30 July 2017

Academic Editor: Shi-Hai Dong

Copyright ( 2017 G. P. Singh and Binaya K. Bishi. This is an open access article distributed under the Creative Commons Attribution License, which permits unrestricted use, distribution, and reproduction in any medium, provided the original work is properly cited. The publication of this article was funded by SCOAP ${ }^{3}$.

\begin{abstract}
We have presented FRW cosmological model in the framework of Brans-Dicke theory. This paper deals with a new proposed form of deceleration parameter and cosmological constant $\Lambda$. The effect of bulk viscosity is also studied in the presence of modified Chaplygin gas equation of state $\left(p=A \rho-B / \rho^{n}\right)$. Furthermore, we have discussed the physical behaviours of the models.
\end{abstract}

\section{Introduction}

It has been well established that alternative theories of gravitation played an important role in understanding the models of the Universe. For the last few decades, researchers have shown more interest in alternative theories of gravitation especially scalar-tensor theories of gravity. The Brans-Dicke theory (BDT) of gravity is the one of the most successful alternative theories among all alternative theories of gravitation. This theory is consisting of a massless scalar field $\phi$ and a dimensionless constant $\omega$ describing the strength of the coupling between $\phi$ and the matter [1]. In the BDT, gravitational constant $G$ is treated as the reciprocal of a massless scalar field $\phi$, where $\phi$ is expected to satisfy scalar wave equations and its source is all matter in the Universe.

In a pioneering work, both research contributions by Mathiazhagan \& Johri [2] and later La \& Steinhardt [3] showed that the idea of inflationary expansion with a firstorder phase transition can be made to work more satisfactorily if one considers the BDT in place of general relativity. The interesting consequence of $\mathrm{BD}$ scalar field is that the modified field equations would express the scale factor $R(t)$ as a power function of time and not as an exponential function, so that one attains the so-called "graceful exit" from the inflationary vacuum phase through a first-order phase transition. Hyperextend inflation [4] generalizes the results of extended inflation in BDT and solves the graceful exit problem in a natural way, without recourse to any finetuning as required in relativistic models. Romero \& Barros [5] discussed the limit of the Brans-Dicke theory of gravity when $\omega \rightarrow \infty$ and showed by examples that, in this limit, it is not always true that BDT reduces to general relativity. From the literature, it is known that the result of BDT is close to Einstein theory of general relativity for large value of the coupling parameter $(\omega \geq 500)[6,7]$. A more recent bound on the Brans-Dicke parameter $\omega$ is $\omega>3300$ [7]. A number of researchers [8-15] have discussed various aspects of expanding cosmological models in BDT.

Cosmological observations $[16,17]$ and various related research clearly indicate that the constituent of the present Universe is dominated by dark energy, which constitutes about three-fourths of the whole matter of our Universe. There are several candidates for dark energy like quintessence, phantom, quintom, holographic dark energy, K-essence, Chaplygin gas, and cosmological constant. Among all the dark energy candidates, cosmological constant is the more favoured. It provides enough negative pressure to account for the acceleration and contributes an energy density of same order of magnitude compared to the energy density of the matter [18]. The discrepancy of observed value 
and theoretical value of cosmological constant is usually referred as cosmological constant problem in literature. This problem is the puzzling problem in standard cosmology. The cosmological constant bears a dynamical decaying character so that it might be large at early epoch and approaching to a small value at the present epoch.

The effect of cosmological constant has been discussed in the literature in the context of general relativity and its alternative theories. T. Singh and T. Singh [19] presented a cosmological model in BDT by considering cosmological constant as a function of scalar field $\phi$. Exact cosmological solutions in BDT with uniform cosmological "constant" have been studied by Pimentel [20]. A class of flat FRW cosmological models with cosmological "constant" in BDT have also been obtained by Ahmadi-Azar \& Riazi [21]. The age of the Universe from a view point of the nucleosynthesis with $\Lambda$ term in BDT was investigated by Etoh et al. [22]. Azad \& Islam [23] extended the idea of T. Singh and T. Singh [19] to study cosmological constant in Bianchi type I modified Brans-Dicke cosmology. Qiang et al. [24] discussed cosmic acceleration in five-dimensional BDT using interacting Higgs and Brans-Dicke fields. Smolyakov [25] investigated a model which provides the necessary value of effective cosmological "constant" at the classical level. Recently, embedding general relativity with varying cosmological term in five-dimensional BDT of gravity in vacuum has been discussed by Reyes \& Aguilar [26]. Singh et al. [27] have studied the dynamic cosmological constant in BDT.

On the other side, it is known from the literature that for early evolution of the Universe, bulk viscosity is supposed to play a very important role. The presence of viscosity in the fluid explores many dynamics of the homogeneous cosmological models. The bulk viscosity coefficient determines the magnitude of the viscous stress relative to the expansion. Recently Saadat \& Pourhassan [28] investigated the FRW bulk viscous cosmology with modified cosmic Chaplygin gas. Many researchers also have shown interest in FRW bulk viscous cosmological models in different contexts (see Saadat \& Pourhassan [28] and references therein).

Motivated by the above studies, here we have discussed the variable cosmological constant $\Lambda$ for FRW metric in the context of BDT with a special form of deceleration parameter.

\section{Field Equations}

The field equation of Brans-Dicke theory in presence of cosmological constant may be written as

$$
\begin{aligned}
& G_{i j}-\Lambda g_{i j}+\frac{\omega}{\phi^{2}}\left[\phi_{; i} \phi_{; j}-\frac{1}{2} g_{i j} \phi_{; k} \phi^{; k}\right] \\
& +\frac{1}{\phi}\left[\phi_{; i ; j}-g_{i j} \square \phi\right]=\frac{8 \pi}{\phi} T_{i j}, \\
& \square \phi=\phi_{; i}^{; i}=\frac{8 \pi}{2 \omega+3} T_{, i}^{i},
\end{aligned}
$$

where $\phi$ is the scalar field. The energy-momentum tensor $T_{i j}$ of the cosmic fluid in the presence of bulk viscosity may be be defined as

$$
T_{i j}=(\rho+p+\Pi) u_{i} u_{j}-(p+\Pi) g_{i j} .
$$

Let us consider a homogeneous and isotropic Universe represented by FRW space-time metric as

$$
d s^{2}=d t^{2}-R^{2}(t)\left[\frac{d r^{2}}{1-k r^{2}}+r^{2}\left(d \theta^{2}+\sin ^{2} \theta d \phi^{2}\right)\right],
$$

where $k(=1,0,-1)$ is the curvature parameter, which represents closed, flat, and open model of the Universe and $R(t)$ is the scale factor.

The FRW metric (3) and energy-momentum tensor (2) along with Brans-Dicke field equations yield the following equations:

$$
\begin{aligned}
& 3\left(\frac{\dot{R}}{R}\right)^{2}+3 \frac{\dot{R} \dot{\phi}}{R \phi}-\frac{\omega}{2}\left(\frac{\dot{\phi}}{\phi}\right)^{2}+3 \frac{k}{R^{2}}=\frac{8 \pi}{\phi} \rho+\Lambda \\
& 2 \frac{\ddot{R}}{R}+\left(\frac{\dot{R}}{R}\right)^{2}+\frac{\ddot{\phi}}{\phi}+\frac{\omega}{2}\left(\frac{\dot{\phi}}{\phi}\right)^{2}+2 \frac{\dot{R} \dot{\phi}}{R \phi}+\frac{k}{R^{2}} \\
& =\frac{-8 \pi}{\phi}(p+\Pi)+\Lambda \\
& \frac{\ddot{\phi}}{\phi}+3 \frac{\dot{R} \dot{\phi}}{R \phi}=\frac{8 \pi}{\phi} \frac{\rho-3 p-3 \Pi}{3+2 \omega}+\frac{2 \Lambda}{3+2 \omega} .
\end{aligned}
$$

\section{Solution of the Field Equations}

In order to find exact solutions of basic field equations (4), one must ensure that set of equations should be closed. Thus, two more physically reasonable relations are required among the variables.

First we consider a well accepted power law relation between scale factor $R(t)$ and scalar field $\phi$ of the form [27]

$$
\phi=\phi_{0} R^{\alpha_{1}}
$$

and as it has been well established the expansion of present Universe is accelerating. In order to study a cosmological model with early deceleration and late time acceleration, we have proposed deceleration parameter of the form

$$
q=\frac{\alpha_{2}+\alpha_{3} t}{1+t}
$$

as the second physically plausible relation, where $\alpha_{2}, \alpha_{3} \in \mathbb{R}$. The considered form of deceleration parameter is motivated by the bilinear form of deceleration parameter, Mishra \& Chand [29]. Deceleration parameter is useful to classify the models of the Universe. From literature we know that deceleration parameter is a constant quantity or it depends on time. In the case when rate of expansion never changes and $\dot{R}$ is constant, the scaling factor is proportional to 
time, which leads to zero deceleration. In case when $H$ is constant, the deceleration parameter $(q)$ is also constant $(-1)$. In de-Sitter and steady state Universe such cases arises. Now we will classify the cosmological models on the basis of time dependence on Hubble parameter and deceleration parameter as follows, Bolotin et al. [30].

(i) $H>0, q>0$ : expanding and decelerating;

(ii) $H>0, q<0$ : expanding and accelerating;

(iii) $H<0, q>0$ : contracting and decelerating;

(iv) $H<0, q<0$ : contracting and accelerating;

(v) $H>0, q=0$ : expanding, zero deceleration/constant expansion;

(vi) $H<0, q=0$ : contracting, zero deceleration;

(vii) $H=0, q=0$ : static.

From the above classification, (i), (ii), and (v) are possible cases as in the present scenario our Universe is expanding. Again also we have found the following type of expansion exhibit by our Universe.

(i) $q<-1$ : superexponential expansion;

(ii) $-1 \leq q<0$ : exponential expansion (for $q=-1$ known as de-Sitter expansion);

(iii) $q=0$ : expansion with constant rate;

(iv) $-1<q<1$ : accelerating power expansion;

(v) $q>0$ : decelerating expansion.

We consider third physically plausible relation as the modified Chaplygin gas equation of state as follows [31, 32]:

$$
p=A \rho-\frac{B}{\rho^{n}}
$$

where $A>0, B>0$ are constants and $0 \leq n \leq 1$.
The set of field equations (4) with the help of (5) may be written as

$$
\begin{aligned}
& \left(\frac{6+6 \alpha_{1}-\omega \alpha_{1}^{2}}{2}\right)\left(\frac{\dot{R}}{R}\right)^{2}+\frac{3 k}{R^{2}}=\frac{8 \pi}{\phi_{0} R^{\alpha_{1}}} \rho+\Lambda \\
& \left(2+\alpha_{1}\right) \frac{\ddot{R}}{R}+\left(\frac{2+2 \alpha_{1}+2 \alpha_{1}^{2}+\omega \alpha_{1}^{2}}{2}\right)\left(\frac{\dot{R}}{R}\right)^{2}+\frac{k}{R^{2}} \\
& =\frac{-8 \pi}{\phi_{0} R^{\alpha_{1}}}(p+\Pi)+\Lambda \\
& {\left[\alpha_{1} \frac{\ddot{R}}{R}+\alpha_{1}\left(\alpha_{1}+2\right)\left(\frac{\dot{R}}{R}\right)^{2}\right](3+2 \omega)} \\
& =\frac{8 \pi}{\phi_{0} R^{\alpha_{1}}}(\rho-3 p-3 \Pi)+2 \Lambda \text {. }
\end{aligned}
$$

Equations (8) leads us to

$$
2\left(3-\omega \alpha_{1}\right) \frac{\ddot{R}}{R}+\left(6-4 \omega \alpha_{1}-\omega \alpha_{1}^{2}\right)\left(\frac{\dot{R}}{R}\right)^{2}+\frac{6 k}{R^{2}}=2 \Lambda .
$$

This equation is useful for obtaining the various cosmological solutions.

Now our problem is to evaluate the $R(t)$, which is obtained from the relation

$$
-\frac{\dot{H}}{H^{2}}=1+q
$$

With the help of (6) and integrating (10), we obtained

$$
H=\frac{1}{\left(1+\alpha_{3}\right) t+\left(\alpha_{2}-\alpha_{3}\right) \ln (1+t)+c_{1}},
$$

where $c_{1}$ is a constant of integration. The condition $H \rightarrow \infty$ when $t \rightarrow 0$ yields $c_{1}=0$. Thus, (11) takes the form

$$
H=\frac{1}{\left(1+\alpha_{3}\right) t+\left(\alpha_{2}-\alpha_{3}\right) \ln (1+t)} .
$$

Equation (12) is expressed as

$$
\begin{aligned}
H & =\frac{1}{\left(1+\alpha_{3}\right) t+\left(\alpha_{2}-\alpha_{3}\right)\left[t-t^{2} / 2+t^{3} / 3-t^{4} / 4+t^{5} / 5-t^{6} / 6+t^{7} / 7-\cdots\right]} \\
& =\frac{1}{\left(1+\alpha_{2}\right) t+\left(\alpha_{2}-\alpha_{3}\right)\left[-t^{2} / 2+t^{3} / 3-t^{4} / 4+t^{5} / 5-t^{6} / 6+t^{7} / 7-\cdots\right]}=\frac{1}{\left(1+\alpha_{2}\right) t}\{1 \\
& \left.-\frac{\left(\alpha_{2}-\alpha_{3}\right)}{1+\alpha_{2}}\left[\frac{t}{2}-\frac{t^{2}}{3}+\frac{t^{3}}{4}-\frac{t^{4}}{5}+\frac{t^{5}}{6}-\frac{t^{6}}{7}+\cdots\right]\right\}^{-1}=\frac{1}{\left(1+\alpha_{2}\right) t}[1 \\
& +\left(\frac{\alpha_{2}-\alpha_{3}}{1+\alpha_{2}}\right)\left(\frac{t}{2}-\frac{t^{2}}{3}+\frac{t^{3}}{4}-\frac{t^{4}}{5}+\frac{t^{5}}{6}-\frac{t^{6}}{7}+\cdots\right)+\left(\frac{\alpha_{2}-\alpha_{3}}{1+\alpha_{2}}\right)^{2}\left(\frac{t}{2}-\frac{t^{2}}{3}+\frac{t^{3}}{4}-\frac{t^{4}}{5}+\frac{t^{5}}{6}-\frac{t^{6}}{7}+\cdots\right)^{2} \\
& \left.+\left(\frac{\alpha_{2}-\alpha_{3}}{1+\alpha_{2}}\right)^{3}\left(\frac{t}{2}-\frac{t^{2}}{3}+\frac{t^{3}}{4}-\frac{t^{4}}{5}+\frac{t^{5}}{6}-\frac{t^{6}}{7}+\cdots\right)^{3}+\left(\frac{\alpha_{2}-\alpha_{3}}{1+\alpha_{2}}\right)^{4}\left(\frac{t}{2}-\frac{t^{2}}{3}+\frac{t^{3}}{4}-\frac{t^{4}}{5}+\frac{t^{5}}{6}-\frac{t^{6}}{7}+\cdots\right)^{4}+\cdots\right]
\end{aligned}
$$


Simplifying the above expression we obtained

$$
\begin{aligned}
H= & \frac{1}{\left(1+\alpha_{2}\right) t}+k_{0}+k_{1} t+k_{2} t^{2}+k_{3} t^{3}+k_{4} t^{4} \\
& +O\left(t^{5}\right)
\end{aligned}
$$

where

$$
\begin{aligned}
k_{0}= & \frac{\alpha_{2}-\alpha_{3}}{2\left(1+\alpha_{2}\right)^{2}} \\
k_{1}= & \frac{1}{1+\alpha_{2}}\left[\frac{\left(\alpha_{2}-\alpha_{3}\right)^{2}}{4\left(1+\alpha_{2}\right)^{2}}-\frac{\left(\alpha_{2}-\alpha_{3}\right)}{3\left(1+\alpha_{2}\right)}\right] \\
k_{2}= & \frac{1}{1+\alpha_{2}}\left[\frac{\alpha_{2}-\alpha_{3}}{4\left(1+\alpha_{2}\right)}-\frac{\left(\alpha_{2}-\alpha_{3}\right)^{2}}{3\left(1+\alpha_{2}\right)^{2}}\right. \\
+ & \left.\frac{\left(\alpha_{2}-\alpha_{3}\right)^{3}}{8\left(1+\alpha_{2}\right)^{3}}\right] \\
k_{3} & =\frac{1}{1+\alpha_{2}}\left[-\frac{\alpha_{2}-\alpha_{3}}{5\left(1+\alpha_{2}\right)}+\frac{13\left(\alpha_{2}-\alpha_{3}\right)^{2}}{36\left(1+\alpha_{2}\right)^{2}}\right.
\end{aligned}
$$

$$
\begin{aligned}
- & \left.\frac{\left(\alpha_{2}-\alpha_{3}\right)^{3}}{4\left(1+\alpha_{2}\right)^{3}}+\frac{\left(\alpha_{2}-\alpha_{3}\right)^{4}}{16\left(1+\alpha_{2}\right)^{4}}\right] \\
k_{4} & =\frac{1}{1+\alpha_{2}}\left[\frac{\alpha_{2}-\alpha_{3}}{6\left(1+\alpha_{2}\right)}-\frac{11\left(\alpha_{2}-\alpha_{3}\right)^{2}}{30\left(1+\alpha_{2}\right)^{2}}\right. \\
+ & \left.\frac{17\left(\alpha_{2}-\alpha_{3}\right)^{3}}{48\left(1+\alpha_{2}\right)^{3}}-\frac{\left(\alpha_{2}-\alpha_{3}\right)^{4}}{6\left(1+\alpha_{2}\right)^{4}}\right] .
\end{aligned}
$$

Integration of (14) leads us to

$$
R=c_{2} t^{1 /\left(1+\alpha_{2}\right)} e^{T_{1}(t)}
$$

where $T_{1}(t)=k_{0} t+k_{1}\left(t^{2} / 2\right)+k_{2}\left(t^{3} / 3\right)+k_{3}\left(t^{4} / 4\right)+k_{4}\left(t^{5} / 5\right)+$ $O\left(t^{6}\right)$. The solutions of the field equation (8) are expressed as follows: the energy density $\rho$ is obtained as

$$
\rho=\frac{k_{5} t^{\alpha_{1} /\left(1+\alpha_{2}\right)} e^{\alpha_{1} T_{1}(t)}\left(\rho_{1}+\rho_{2} t\right)}{(1+t)\left[\left(1+\alpha_{3}\right) t+\left(\alpha_{2}-\alpha_{3}\right) \ln (1+t)\right]^{2}}
$$

where $k_{5}=\phi_{0} c_{2}^{\alpha_{1}} / 8 \pi, \rho_{1}=\left(3+2 w-w \alpha_{2}\right) \alpha_{1}+3 \alpha_{2}, \rho_{2}=$ $\left(3+2 w-w \alpha_{3}\right) \alpha_{1}+3 \alpha_{3}$.

The pressure $p$ is given as

$$
p=\frac{A k_{5}^{n+1} t^{(n+1) \alpha_{1} /\left(1+\alpha_{2}\right)} e^{(n+1) \alpha_{1} T_{1}(t)}\left(\rho_{1}+\rho_{2} t\right)^{n+1}-B(1+t)^{n+1}\left[\left(1+\alpha_{3}\right) t+\left(\alpha_{2}-\alpha_{3}\right) \ln (1+t)\right]^{2 n+2}}{k_{5}^{n} t^{n \alpha_{1} /\left(1+\alpha_{2}\right)} e^{n \alpha_{1} T_{1}(t)}\left(\rho_{1}+\rho_{2} t\right)^{n}(1+t)\left[\left(1+\alpha_{3}\right) t+\left(\alpha_{2}-\alpha_{3}\right) \ln (1+t)\right]^{2}} .
$$

The bulk viscous stress $\Pi$ is expressed as

$$
\begin{gathered}
k_{5}^{n+1} t^{(n+1) \alpha_{1} /\left(1+\alpha_{2}\right)} e^{(n+1) \alpha_{1} T_{1}(t)}\left[\frac{\rho_{1}+\rho_{2} t}{(1+t)\left[\left(1+\alpha_{3}\right) t+\left(\alpha_{2}-\alpha_{3}\right) \ln (1+t)\right]^{2}}\right]^{n} \\
\times \Pi=\frac{\left[\left(\Pi_{1}+\Pi_{2} t\right) /(1+t)\left[\left(1+\alpha_{3}\right) t+\left(\alpha_{2}-\alpha_{3}\right) \ln (1+t)\right]^{2}+2 k / c_{2}^{2} t^{2 /\left(1+\alpha_{2}\right)} e^{2 T_{1}(t)}\right]+B}{k_{5}^{n} t^{n \alpha_{1} /\left(1+\alpha_{2}\right)} e^{n \alpha_{1} T_{1}(t)}\left[\left(\rho_{1}+\rho_{2} t\right) /(1+t)\left[\left(1+\alpha_{3}\right) t+\left(\alpha_{2}-\alpha_{3}\right) \ln (1+t)\right]^{2}\right]^{n}},
\end{gathered}
$$

where $\Pi_{1}=-(1+w) \alpha_{1}^{2}-(1+3 A) \alpha_{2}+\left[\left(\alpha_{2}-2\right)(1+A) w-\right.$ $\left.1-3 A+\alpha_{2}\right] \alpha_{1}+2$ and $\Pi_{2}=-(1+w) \alpha_{1}^{2}-(1+3 A) \alpha_{3}+\left[\left(\alpha_{2}-\right.\right.$ 2) $\left.(1+A) w-1-3 A+\alpha_{3}\right] \alpha_{1}+2$.

The cosmological constant $\Lambda$ is expressed as

$$
\begin{aligned}
\Lambda= & \frac{\Lambda_{1}+\Lambda_{2} t}{(1+t)\left[\left(1+\alpha_{3}\right) t+\left(\alpha_{2}-\alpha_{3}\right) \ln (1+t)\right]^{2}} \\
& +\frac{3 k}{c_{2}^{2} t^{2 /\left(1+\alpha_{2}\right)} e^{2 T_{1}(t)}},
\end{aligned}
$$

where $\Lambda_{1}=-0.5 w \alpha_{1}^{2}+w\left(\alpha_{2}-2\right) \alpha_{1}-3\left(\alpha_{2}-1\right)$ and $\Lambda_{2}=$ $-0.5 w \alpha_{1}^{2}+w\left(\alpha_{3}-2\right) \alpha_{1}-3\left(\alpha_{3}-1\right)$.
Now, let us start with our proposed form of deceleration parameter $q$. The different form of deceleration parameter is evolved as a result of considered value of $\alpha_{2}$ and $\alpha_{3}$, which is expressed in Table 1. We know that in present scenario our Universe is accelerating. Thus serial numbers (2), (5), (8), and (9) of Table 1 exhibit accelerating model. Now we will discuss the deceleration parameter in serial numbers (2), (5), (8), and (9) of Table 1. For the choice of $\alpha_{2}=\alpha=\alpha_{4}$, the deceleration parameter $q$ in serial number (2) and (5) of Table 1 reduces to $q=-\alpha t /(1+t)$ and $q=-\alpha(1+t) /(1+t)$, respectively, which is discussed by Mishra and Chand [29]. They called this deceleration parameter as bilinear variable deceleration parameter. We will discuss the case where $\alpha_{2} \neq \alpha \neq \alpha_{4}$ of serial number 
TABLE 1: Different forms of deceleration parameter depending on the parameters $\alpha_{2}$ and $\alpha_{3}$ and behaviour of cosmological models according to the deceleration parameter $q$.

\begin{tabular}{|c|c|c|c|}
\hline S. number & Possible value of $\alpha_{2}$ and $\alpha_{3}$ & Form of deceleration parameter $q$ & Behaviour of cosmological model \\
\hline \multirow{2}{*}{ (1) } & $\alpha_{2}=0$ & $a=\frac{\alpha_{3} t}{2}$ & \multirow{2}{*}{ Decelerating } \\
\hline & $\alpha_{3}>0$ & $4-\overline{1+t}$ & \\
\hline \multirow{2}{*}{ (2) } & $\alpha_{2}=0$ & $a=-\frac{\alpha_{4} t}{2}$ & \multirow{2}{*}{ Accelerating } \\
\hline & $\alpha_{3}<0\left(\alpha_{3}=-\alpha_{4}, \alpha_{4}>0\right)$ & $1+t$ & \\
\hline \multirow[t]{2}{*}{ (3) } & $\alpha_{2}=0$ & $q=0$ & \multirow{2}{*}{ Expansion with constant rate } \\
\hline & $\alpha_{3}=0$ & & \\
\hline \multirow{2}{*}{ (4) } & $\alpha_{2}>0$ & $a_{2}=\alpha_{2}+\alpha_{3} t$ & \multirow{2}{*}{ Decelerating } \\
\hline & $\alpha_{3}>0$ & $4-\frac{1+t}{1+2}$ & \\
\hline \multirow[t]{2}{*}{ (5) } & $\alpha_{2}>0$ & $a=\frac{\alpha_{2}-\alpha_{4} t}{2}$ & \multirow{2}{*}{ Phase transition from decelerating to accelerating } \\
\hline & $\alpha_{3}<0$ & $1-1+t$ & \\
\hline \multirow{2}{*}{ (6) } & $\alpha_{2}>0$ & $q=\frac{\alpha_{2}}{2}$ & \multirow{2}{*}{ Decelerating } \\
\hline & $\alpha_{3}=0$ & $1+t$ & \\
\hline \multirow[t]{2}{*}{ (7) } & $\alpha_{2}<0\left(\alpha_{2}=-\alpha_{5}, \alpha_{5}>0\right)$ & $a=\frac{-\alpha_{5}+\alpha_{3} t}{}$ & \multirow{2}{*}{ Phase transition from accelerating to decelerating } \\
\hline & $\alpha_{3}>0$ & $1+t$ & \\
\hline \multirow{2}{*}{ (8) } & $\alpha_{2}<0$ & $a=-\alpha_{5}-\alpha_{4} t$ & \multirow{2}{*}{ Accelerating } \\
\hline & $\alpha_{3}<0$ & $q-\overline{1+t}$ & \\
\hline \multirow[t]{2}{*}{ (9) } & $\alpha_{2}<0$ & $\alpha_{5}$ & \multirow{2}{*}{ Accelerating } \\
\hline & $\alpha_{3}=0$ & $1+t$ & \\
\hline
\end{tabular}

(5) of Table 1 and also serial numbers (8) and (9) of Table 1. According to the serial numbers (5), (8), and (9) of Table 1 we have three different models, which are discussed below.

3.1. Model I. The deceleration parameter $q$ in (6) for $\alpha_{2}>0$ and $\alpha_{3}<0$ takes the form

$$
q=\frac{\alpha_{2}-\alpha_{4} t}{1+t}, \quad \alpha_{3}=-\alpha_{4}, \alpha_{4}>0
$$

Here we noticed that $q>0$ for $0<t<\alpha_{2} / \alpha_{4}$ and $q<0$ for $t>\alpha_{2} / \alpha_{4}$, which means that our Universe is decelerating and accelerating in the provided ranges, respectively. Thus our Universe undergoes a phase transition from decelerating to accelerating phase.

For model I, the physical parameters are obtained as follows.

The Hubble parameter in (12) takes the form

$$
H=\frac{1}{\left(1-\alpha_{4}\right) t+\left(\alpha_{2}+\alpha_{4}\right) \ln (1+t)} .
$$

The scale factor $R(t)$ in (16) is expressed as

$$
R=c_{2} t^{1 /\left(1+\alpha_{2}\right)} e^{T_{1}(t)}
$$

where $T_{1}(t)=k_{0} t+k_{1}\left(t^{2} / 2\right)+k_{2}\left(t^{3} / 3\right)+k_{3}\left(t^{4} / 4\right)+k_{4}\left(t^{5} / 5\right)+$ $O\left(t^{6}\right)$. And

$$
k_{0}=\frac{\alpha_{2}+\alpha_{4}}{2\left(1+\alpha_{2}\right)^{2}}
$$

$$
\begin{aligned}
k_{1} & =\frac{1}{1+\alpha_{2}}\left[\frac{\left(\alpha_{2}+\alpha_{4}\right)^{2}}{4\left(1+\alpha_{2}\right)^{2}}-\frac{\left(\alpha_{2}+\alpha_{4}\right)}{3\left(1+\alpha_{2}\right)}\right] \\
k_{2} & =\frac{1}{1+\alpha_{2}}\left[\frac{\alpha_{2}+\alpha_{4}}{4\left(1+\alpha_{2}\right)}-\frac{\left(\alpha_{2}+\alpha_{4}\right)^{2}}{3\left(1+\alpha_{2}\right)^{2}}\right. \\
& \left.+\frac{\left(\alpha_{2}+\alpha_{4}\right)^{3}}{8\left(1+\alpha_{2}\right)^{3}}\right] \\
k_{3}= & \frac{1}{1+\alpha_{2}}\left[-\frac{\alpha_{2}+\alpha_{4}}{5\left(1+\alpha_{2}\right)}+\frac{13\left(\alpha_{2}+\alpha_{4}\right)^{2}}{36\left(1+\alpha_{2}\right)^{2}}\right. \\
& -\frac{\left(\alpha_{2}+\alpha_{4}\right)^{3}}{\left.4\left(1+\alpha_{2}\right)^{3}+\frac{\left(\alpha_{2}+\alpha_{4}\right)^{4}}{16\left(1+\alpha_{2}\right)^{4}}\right]} \\
k_{4}= & \frac{1}{1+\alpha_{2}}\left[\frac{\alpha_{2}+\alpha_{4}}{6\left(1+\alpha_{2}\right)}-\frac{11\left(\alpha_{2}+\alpha_{4}\right)^{2}}{30\left(1+\alpha_{2}\right)^{2}}\right. \\
+ & \left.\frac{17\left(\alpha_{2}+\alpha_{4}\right)^{3}}{48\left(1+\alpha_{2}\right)^{3}}-\frac{\left(\alpha_{2}+\alpha_{4}\right)^{4}}{6\left(1+\alpha_{2}\right)^{4}}\right] .
\end{aligned}
$$

The FRW space-time metric in (3) takes the form

$$
\begin{aligned}
d s^{2} & =d t^{2}-c_{2}^{2} t^{2 /\left(1+\alpha_{2}\right)} e^{2 T_{1}(t)}\left[\frac{d r^{2}}{1-k r^{2}}\right. \\
& \left.+r^{2}\left(d \theta^{2}+\sin ^{2} \theta d \phi^{2}\right)\right]
\end{aligned}
$$


with the above mentation $k_{i},(i=0,1,2,3,4)$. The energy density $(\rho)$, pressure $(p)$, bulk viscous stress $(\Pi)$, and cosmological constant $(\Lambda)$ in (17), (18), (19), and (20) are expressed as

$$
\rho=\frac{k_{5} t^{\alpha_{1} /\left(1+\alpha_{2}\right)} e^{\alpha_{1} T_{1}(t)}\left(\rho_{1}+\rho_{2} t\right)}{(1+t)\left[\left(1-\alpha_{4}\right) t+\left(\alpha_{2}+\alpha_{4}\right) \ln (1+t)\right]^{2}},
$$

where $k_{5}=\phi_{0} c_{2}^{\alpha_{1}} / 8 \pi, \rho_{1}=\left(3+2 w-w \alpha_{2}\right) \alpha_{1}+3 \alpha_{2}, \rho_{2}=$ $\left(3+2 w+w \alpha_{4}\right) \alpha_{1}-3 \alpha_{4}$.

$$
\begin{gathered}
p=\frac{A k_{5}^{n+1} t^{(n+1) \alpha_{1} /\left(1+\alpha_{2}\right)} e^{(n+1) \alpha_{1} T_{1}(t)}\left(\rho_{1}+\rho_{2} t\right)^{n+1}-B(1+t)^{n+1}\left[\left(1-\alpha_{4}\right) t+\left(\alpha_{2}+\alpha_{4}\right) \ln (1+t)\right]^{2 n+2}}{k_{5}^{n} t^{n \alpha_{1} /\left(1+\alpha_{2}\right)} e^{n \alpha_{1} T_{1}(t)}\left(\rho_{1}+\rho_{2} t\right)^{n}(1+t)\left[\left(1-\alpha_{4}\right) t+\left(\alpha_{2}+\alpha_{4}\right) \ln (1+t)\right]^{2}}, \\
k_{5}^{n+1} t^{(n+1) \alpha_{1} /\left(1+\alpha_{2}\right)} e^{(n+1) \alpha_{1} T_{1}(t)}\left[\frac{\rho_{1}+\rho_{2} t}{(1+t)\left[\left(1-\alpha_{4}\right) t+\left(\alpha_{2}+\alpha_{4}\right) \ln (1+t)\right]^{2}}\right]^{2} \\
\times \Pi=\frac{\left[\left(\Pi_{1}+\Pi_{2} t\right) /(1+t)\left[\left(1-\alpha_{4}\right) t+\left(\alpha_{2}+\alpha_{4}\right) \ln (1+t)\right]^{2}+2 k / c_{2}^{2} t^{2 /\left(1+\alpha_{2}\right)} e^{2 T_{1}(t)}\right]+B}{k_{5}^{n} t^{n \alpha_{1} /\left(1+\alpha_{2}\right)} e^{n \alpha_{1} T_{1}(t)}\left[\left(\rho_{1}+\rho_{2} t\right) /(1+t)\left[\left(1-\alpha_{4}\right) t+\left(\alpha_{2}+\alpha_{4}\right) \ln (1+t)\right]^{2}\right]^{n}},
\end{gathered}
$$

where $\Pi_{1}=-(1+w) \alpha_{1}^{2}-(1+3 A) \alpha_{2}+\left[\left(\alpha_{2}-2\right)(1+A) w-\right.$ $\left.1-3 A+\alpha_{2}\right] \alpha_{1}+2$ and $\Pi_{2}=-(1+w) \alpha_{1}^{2}+(1+3 A) \alpha_{4}+\left[\left(\alpha_{2}-\right.\right.$ 2) $\left.(1+A) w-1-3 A-\alpha_{4}\right] \alpha_{1}+2$.

$$
\begin{aligned}
\Lambda= & \frac{\Lambda_{1}+\Lambda_{2} t}{(1+t)\left[\left(1-\alpha_{4}\right) t+\left(\alpha_{2}+\alpha_{4}\right) \ln (1+t)\right]^{2}} \\
& +\frac{3 k}{c_{2}^{2} t^{2 /\left(1+\alpha_{2}\right)} e^{2 T_{1}(t)}},
\end{aligned}
$$

where $\Lambda_{1}=-0.5 w \alpha_{1}^{2}+w\left(\alpha_{2}-2\right) \alpha_{1}-3\left(\alpha_{2}-1\right)$ and $\Lambda_{2}=$ $-0.5 w \alpha_{1}^{2}-w\left(\alpha_{4}+2\right) \alpha_{1}+3\left(\alpha_{4}+1\right)$.

Figures 1 and 2 represent the variation of deceleration parameter against time with different values of parameters as presented in the figures for model I. From these figures, we have noticed that when $\alpha_{2}$ is fixed and $\alpha_{4}$ is different and vice versa, deceleration parameter $q$ is a decreasing function of time and it takes values from positive to negative, which shows that our Universe undergoes a phase transition from deceleration phase to acceleration phase. Here we observed that $-1<q<1$ for $0<\alpha_{2} \leq 1$ and $0<\alpha_{4} \leq 1$, which means that within the provided range of $\alpha_{i}(i=2,4)$ our Universe undergoes an accelerating power expansion. It can be observed from Figures 1 and 2 .

The variation of Hubble parameter $H$ and scale factor $R$ against time is plotted in Figures 3 and 4, respectively for model I. As a representative case here we have presented the variation of $H$ and $R$ for fixed $\alpha_{2}=0.5$ and different $\alpha_{4}$ as in figures. It is found that Hubble parameter $H$ is a decreasing function of time and approaching towards zero with the evolution of time. For $0<\alpha_{4} \leq 0.8$ and $\alpha_{5}=0.5$, the scale factor $R$ is an increasing function of time and the higher the value of $\alpha_{4}$, the lower the value of scale factor $R$. For $\alpha_{4} \geq 0.9$ and $\alpha_{2}=0.5$, the scale factor takes a bounce and increases with the evolution of time (see Figure 4).

Figures 5 and 6 represent the variation of energy density $\rho$ and pressure $p$ against time, respectively for model I. From the Figure 5 we pointed out that, in the interval $0<\alpha_{4} \leq$ $0.8 \& \alpha_{2}=0.5$ with the time, energy density decreases for small interval of time and increases to a higher value with the evolution of time. This shows that our Universe is dominated by radiation. For $\alpha_{4} \geq 0.9$ and $\alpha_{2}=0.5$ the energy density is a decreasing function of time and approaches to zero with the evolution of time. In present scenario such type of qualitative behaviour of energy density is observed from observational data. From pressure profile (Figure 6) we observed that, in the intervals $0<\alpha_{4} \leq 0.8$ and $\alpha_{2}=0.5$, the pressure is negative for small interval of time and increases with the evolution of time. In the intervals $0.9 \leq \alpha_{4} \leq 1.2$ and $\alpha_{2}=0.5$, pressure is negative, which follows the observational data but, for $\alpha_{4}>1.2$, it is complex valued; thus we neglect it.

The variation of bulk viscous stress $\Pi$ and cosmological constant $\Lambda$ against time is plotted in Figures 7 and 8 , respectively for model I. Figures indicate the qualitative and quantitative behaviour of both the parameters for open $(k=$ $-1)$, flat $(k=0)$, and closed $(k=1)$ Universe. We have noticed the following points.

\section{Bulk Viscous Stress П (see Figure 7)}

(i) Bulk viscous stress $\Pi$ takes values from positive to negative and approaches to minus infinity $(-\infty)$ with time in case of flat and closed Universe whereas it is negative-positive-negative valued for open Universe in the intervals $0<\alpha_{4} \leq 0.7$ and $\alpha_{2}=0.5$.

(ii) Bulk viscous stress $\Pi$ is positive valued and tends to infinity with the evolution of time for flat and closed Universe whereas it is negative-positive valued for open Universe in $0.7<\alpha_{4} \leq 0.8$ and $\alpha_{2}=0.5$.

(iii) For $\alpha_{4}>0.8$ and $\alpha_{2}=0.5$, bulk viscous stress $\Pi$ is positive valued and tends to infinity with the evolution of time for flat and closed Universe whereas it is negative valued for open Universe.

\section{Cosmological Constant $\Lambda$ (see Figure 8)}

(i) Cosmological constant $\Lambda$ is positive and negative for flat and open Universe and closed Universe, respectively. Cosmological constant $\Lambda \rightarrow 0$ when $t \rightarrow \infty$. 


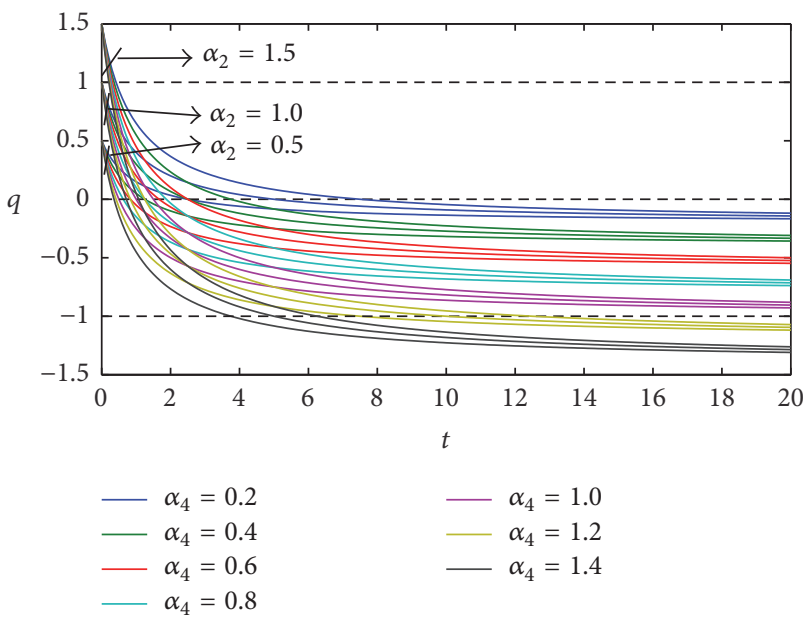

FIGURE 1: Variation of deceleration parameter against time for fixed $\alpha_{2}$ and different $\alpha_{4}$.

(ii) In case of flat and open Universe cosmological constant $\Lambda$ is positive valued for $\alpha_{4}>0.8$ and $\alpha_{2}=0.5$ whereas it is negative valued for open Universe.

(iii) In case of flat Universe cosmological constant $\Lambda \rightarrow 0$ when $t \rightarrow \infty$ but for close and open Universe $\Lambda \rightarrow$ $\infty$ when $t \rightarrow \infty$ and $\Lambda \rightarrow-\infty$ when $t \rightarrow \infty$, respectively.

3.2. Model II. The deceleration parameter $q$ in (6) for $\alpha_{2}<0$ and $\alpha_{3}<0$ takes the form

$$
q=-\frac{\left(\alpha_{5}+\alpha_{4} t\right)}{1+t}, \quad \alpha_{3}=-\alpha_{4}, \alpha_{2}=-\alpha_{5}, \alpha_{4}, \alpha_{5}>0
$$

Here we noticed that $q<0$ for $\alpha_{4}, \alpha_{5}>0$, which means that our Universe is accelerating with the evolution of time.

For model II, the physical parameters are obtained as follows:

The Hubble parameter in (12) takes the form

$$
H=\frac{1}{\left(1-\alpha_{4}\right) t+\left(\alpha_{4}-\alpha_{5}\right) \ln (1+t)} .
$$

The scale factor $R(t)$ in (16) is expressed as

$$
R=c_{2} t^{1 /\left(1-\alpha_{5}\right)} e^{T_{1}(t)}
$$

where $T_{1}(t)=k_{0} t+k_{1}\left(t^{2} / 2\right)+k_{2}\left(t^{3} / 3\right)+k_{3}\left(t^{4} / 4\right)+k_{4}\left(t^{5} / 5\right)+$ $O\left(t^{6}\right)$. And

$$
\begin{aligned}
& k_{0}= \frac{\alpha_{4}-\alpha_{5}}{2\left(1-\alpha_{5}\right)^{2}} \\
& k_{1}= \frac{1}{1-\alpha_{5}}\left[\frac{\left(\alpha_{4}-\alpha_{5}\right)^{2}}{4\left(1-\alpha_{5}\right)^{2}}-\frac{\left(\alpha_{4}-\alpha_{5}\right)}{3\left(1-\alpha_{5}\right)}\right] \\
& k_{2}= \frac{1}{1-\alpha_{5}}\left[\frac{\alpha_{4}-\alpha_{5}}{4\left(1-\alpha_{5}\right)}-\frac{\left(\alpha_{4}-\alpha_{5}\right)^{2}}{3\left(1-\alpha_{5}\right)^{2}}\right. \\
&+\left.\frac{\left(\alpha_{4}-\alpha_{5}\right)^{3}}{8\left(1-\alpha_{5}\right)^{3}}\right] \\
& k_{3}= \frac{1}{1-\alpha_{5}}\left[-\frac{\alpha_{4}-\alpha_{5}}{5\left(1-\alpha_{5}\right)}+\frac{13\left(\alpha_{4}-\alpha_{5}\right)^{2}}{36\left(1-\alpha_{5}\right)^{2}}\right. \\
&\left.-\frac{\left(\alpha_{4}-\alpha_{5}\right)^{3}}{4\left(1-\alpha_{5}\right)^{3}}+\frac{\left(\alpha_{4}-\alpha_{5}\right)^{4}}{16\left(1-\alpha_{5}\right)^{4}}\right] \\
&\left.+\frac{17\left(\alpha_{4}-\alpha_{5}\right)^{3}}{48\left(1-\alpha_{5}\right)^{3}}-\frac{\left(\alpha_{4}-\alpha_{5}\right)^{4}}{6\left(1-\alpha_{5}\right)^{4}}\right] . \\
& k_{4}= \frac{\alpha_{4}-\alpha_{5}}{1-\alpha_{5}}-\frac{11\left(\alpha_{4}-\alpha_{5}\right)^{2}}{6\left(1-\alpha_{5}\right)} \\
& 30\left(1-\alpha_{5}\right)^{2}
\end{aligned}
$$

The FRW space-time metric in (3) takes the form

$$
\begin{aligned}
d s^{2} & =d t^{2}-c_{2}^{2} t^{2 /\left(1-\alpha_{5}\right)} e^{2 T_{1}(t)}\left[\frac{d r^{2}}{1-k r^{2}}\right. \\
& \left.+r^{2}\left(d \theta^{2}+\sin ^{2} \theta d \phi^{2}\right)\right]
\end{aligned}
$$

with the above mentation $k_{i},(i=0,1,2,3,4)$. The energy density $(\rho)$, pressure $(p)$, bulk viscous stress $(\Pi)$, and cosmological constant $(\Lambda)$ in (17), (18), (19), and (20) takes the form

$$
\rho=\frac{k_{5} t^{\alpha_{1} /\left(1-\alpha_{5}\right)} e^{\alpha_{1} T_{1}(t)}\left(\rho_{1}+\rho_{2} t\right)}{(1+t)\left[\left(1-\alpha_{4}\right) t+\left(\alpha_{4}-\alpha_{5}\right) \ln (1+t)\right]^{2}},
$$

where $k_{5}=\phi_{0} c_{2}^{\alpha_{1}} / 8 \pi, \rho_{1}=\left(3+2 w+w \alpha_{5}\right) \alpha_{1}-3 \alpha_{5}, \rho_{2}=$ $\left(3+2 w+w \alpha_{4}\right) \alpha_{1}-3 \alpha_{4}$.

$$
\begin{gathered}
\left.p=\frac{A k_{5}^{n+1} t^{(n+1) \alpha_{1} /\left(1-\alpha_{5}\right)} e^{(n+1) \alpha_{1} T_{1}(t)}\left(\rho_{1}+\rho_{2} t\right)^{n+1}-B(1+t)^{n+1}\left[\left(1-\alpha_{4}\right) t+\left(\alpha_{4}-\alpha_{5}\right) \ln (1+t)\right]^{2 n+2}}{k_{5}^{n} t^{n \alpha_{1} /\left(1-\alpha_{5}\right)} e^{n \alpha_{1} T_{1}(t)}\left(\rho_{1}+\rho_{2} t\right)^{n}(1+t)\left[\left(1-\alpha_{4}\right) t+\left(\alpha_{4}-\alpha_{5}\right) \ln (1+t)\right]^{2}}\right]^{n} \\
\left.k_{5}^{n+1} t^{(n+1) \alpha_{1} /\left(1-\alpha_{5}\right)} e^{(n+1) \alpha_{1} T_{1}(t)}\left[\frac{\rho_{1}+\rho_{2} t}{(1+t)\left[\left(1-\alpha_{4}\right) t+\left(\alpha_{4}-\alpha_{5}\right) \ln (1+t)\right]^{2}}\right]^{2}+2 k / c_{2}^{2} t^{2 /\left(1-\alpha_{5}\right)} e^{2 T_{1}(t)}\right]+B \\
\times \Pi=\frac{\left[\left(\Pi_{1}+\Pi_{2} t\right) /(1+t)\left[\left(1-\alpha_{4}\right) t+\left(\alpha_{4}-\alpha_{5}\right) \ln (1+t)\right]^{2}\right.}{k_{5}^{n} t^{n \alpha_{1} /\left(1-\alpha_{5}\right)} e^{n \alpha_{1} T_{1}(t)}\left[\left(\rho_{1}+\rho_{2} t\right) /(1+t)\left[\left(1-\alpha_{4}\right) t+\left(\alpha_{4}-\alpha_{5}\right) \ln (1+t)\right]^{2}\right]^{n}}
\end{gathered}
$$




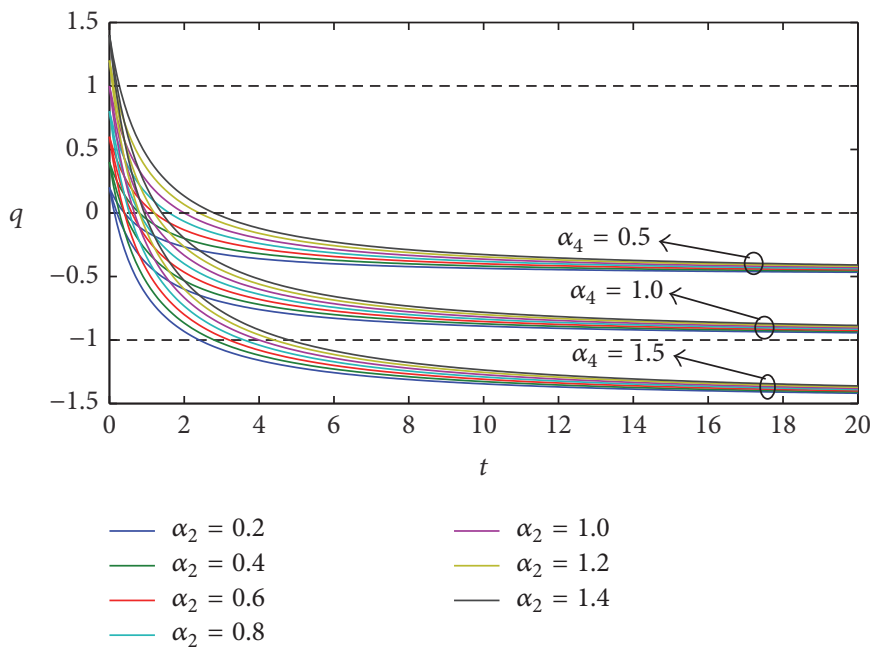

FIGURE 2: Variation of deceleration parameter against time for fixed $\alpha_{4}$ and different $\alpha_{2}$.

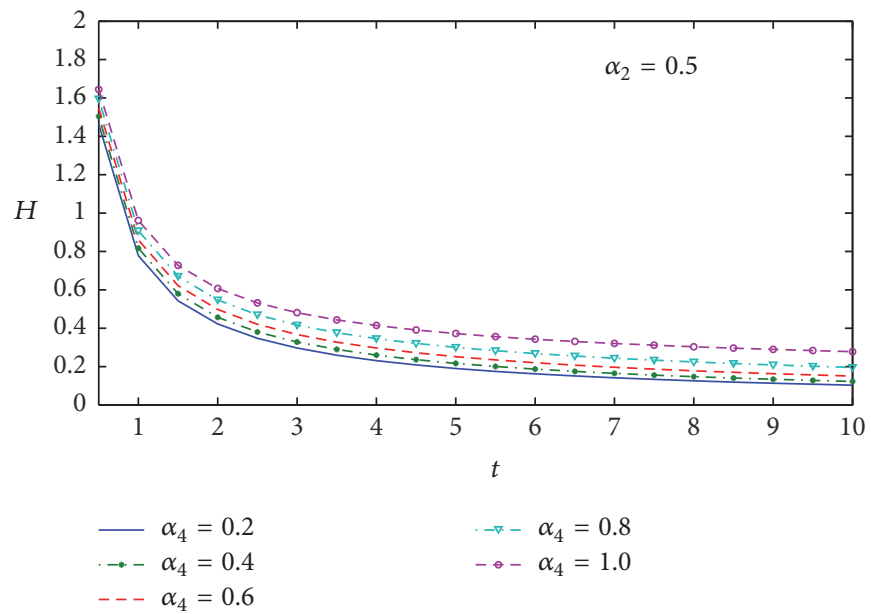

Figure 3: Variation of Hubble parameter against time for fixed $\alpha_{2}=0.5$ and different $\alpha_{4}$.
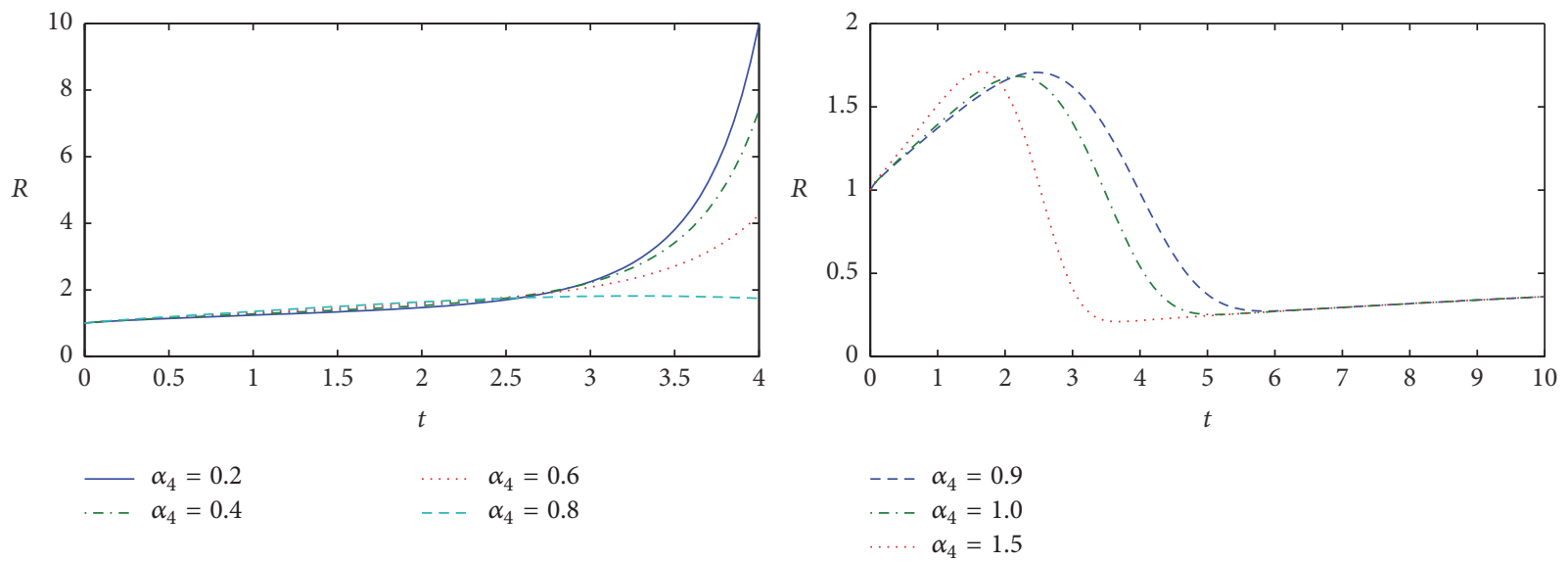

FigURE 4: Variation of scale factor against time for fixed $\alpha_{2}=0.5$ and different $\alpha_{4}$. 

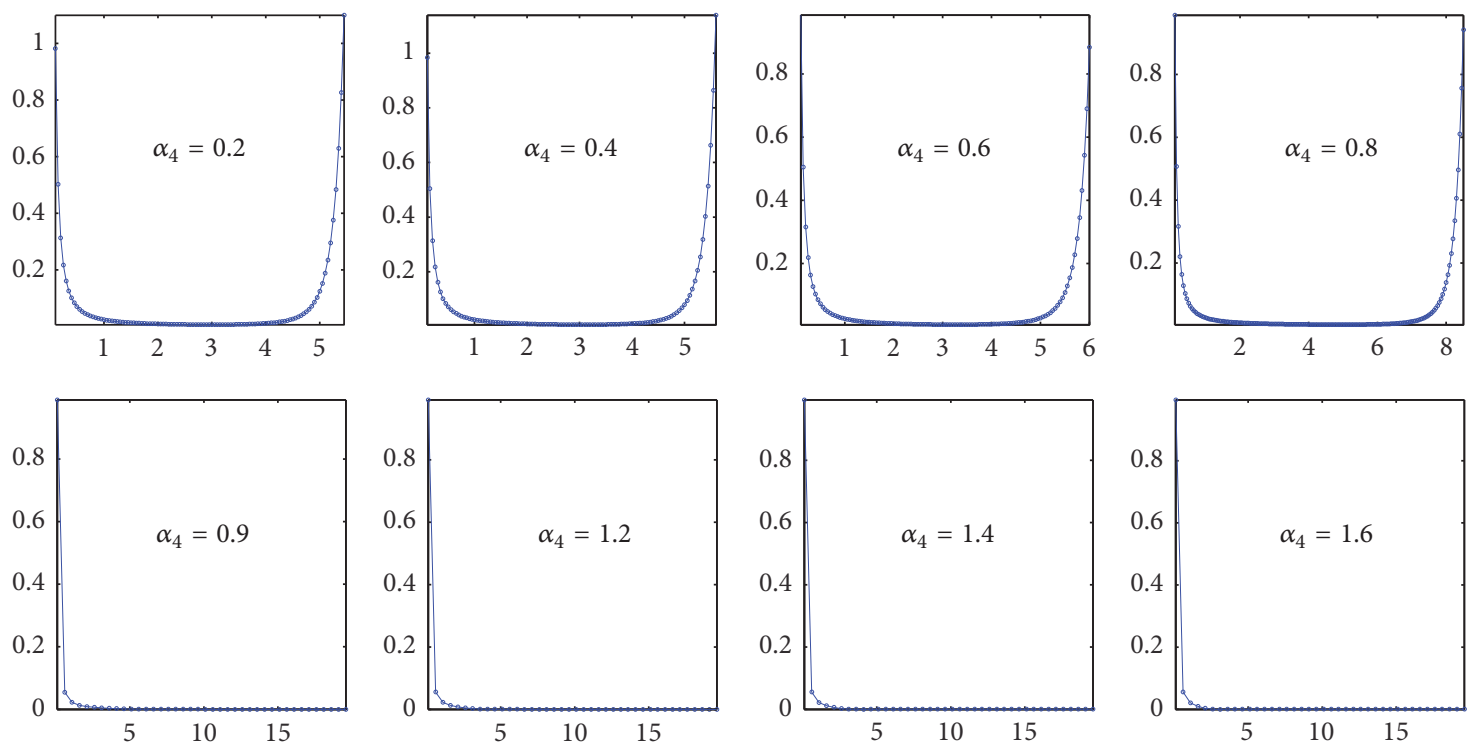

FiguRE 5: Variation of energy density against time for $\alpha_{1}=0.5, \alpha_{2}=0.5, \omega=1, \phi_{0}=1, c_{2}=0.1$, and different $\alpha_{4}$.
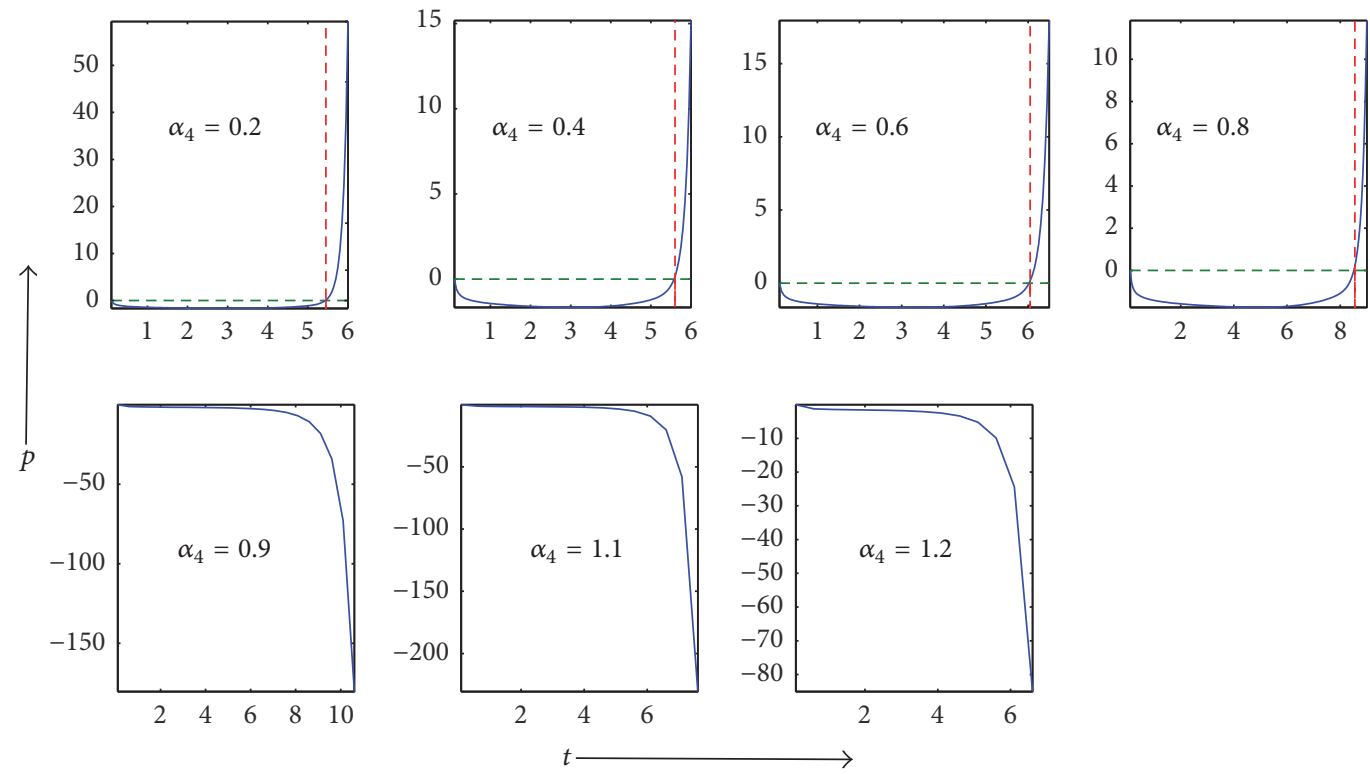

FiguRE 6: Variation of pressure against time for $\alpha_{1}=0.5, \alpha_{2}=0.5, A=B=1, n=0.1, \omega=1, \phi_{0}=1, c_{2}=0.1$, and different $\alpha_{4}$.

where $\Pi_{1}=-(1+w) \alpha_{1}^{2}+(1+3 A) \alpha_{5}-\left[\left(\alpha_{5}+2\right)(1+A) w+\right.$ $\left.1+3 A+\alpha_{5}\right] \alpha_{1}+2$ and $\Pi_{2}=-(1+w) \alpha_{1}^{2}+(1+3 A) \alpha_{4}-\left[\left(\alpha_{5}+\right.\right.$ 2) $\left.(1+A) w+1+3 A+\alpha_{4}\right] \alpha_{1}+2$.

$$
\begin{aligned}
\Lambda= & \frac{\Lambda_{1}+\Lambda_{2} t}{(1+t)\left[\left(1-\alpha_{4}\right) t+\left(\alpha_{4}-\alpha_{5}\right) \ln (1+t)\right]^{2}} \\
& +\frac{3 k}{c_{2}^{2} t^{2 /\left(1-\alpha_{5}\right)} e^{2 T_{1}(t)}}
\end{aligned}
$$

where $\Lambda_{1}=-0.5 w \alpha_{1}^{2}-w\left(\alpha_{5}+2\right) \alpha_{1}+3\left(\alpha_{5}+1\right)$ and $\Lambda_{2}=$ $-0.5 w \alpha_{1}^{2}-w\left(\alpha_{4}+2\right) \alpha_{1}+3\left(\alpha_{4}+1\right)$.

Now we will discuss the physical parameters of the model II. Figures 9 and 10 represent the variation of deceleration parameter against time for fixed $\alpha_{5}$ and different $\alpha_{4}$ and fixed $\alpha_{4}$ and different $\alpha_{5}$, respectively. Here we observed that deceleration parameter is negative and our model is accelerating.

Figures 11, 12, and 13 depict the variation of Hubble parameter $H$ and scale factor $R$ against time, respectively, for model II. The observations are as follows:

(i) Hubble parameter $H$ is a decreasing function of time and tending to zero with the evolution of time. As a representative case, we have considered $\alpha_{5}=0.5$ and different $\alpha_{4}\left(0<\alpha_{4} \leq 1.2\right)$ as in Figure 11 .

(ii) Scale factor increases with the evolution of time. Here we pointed out that the qualitative behaviour of scale 

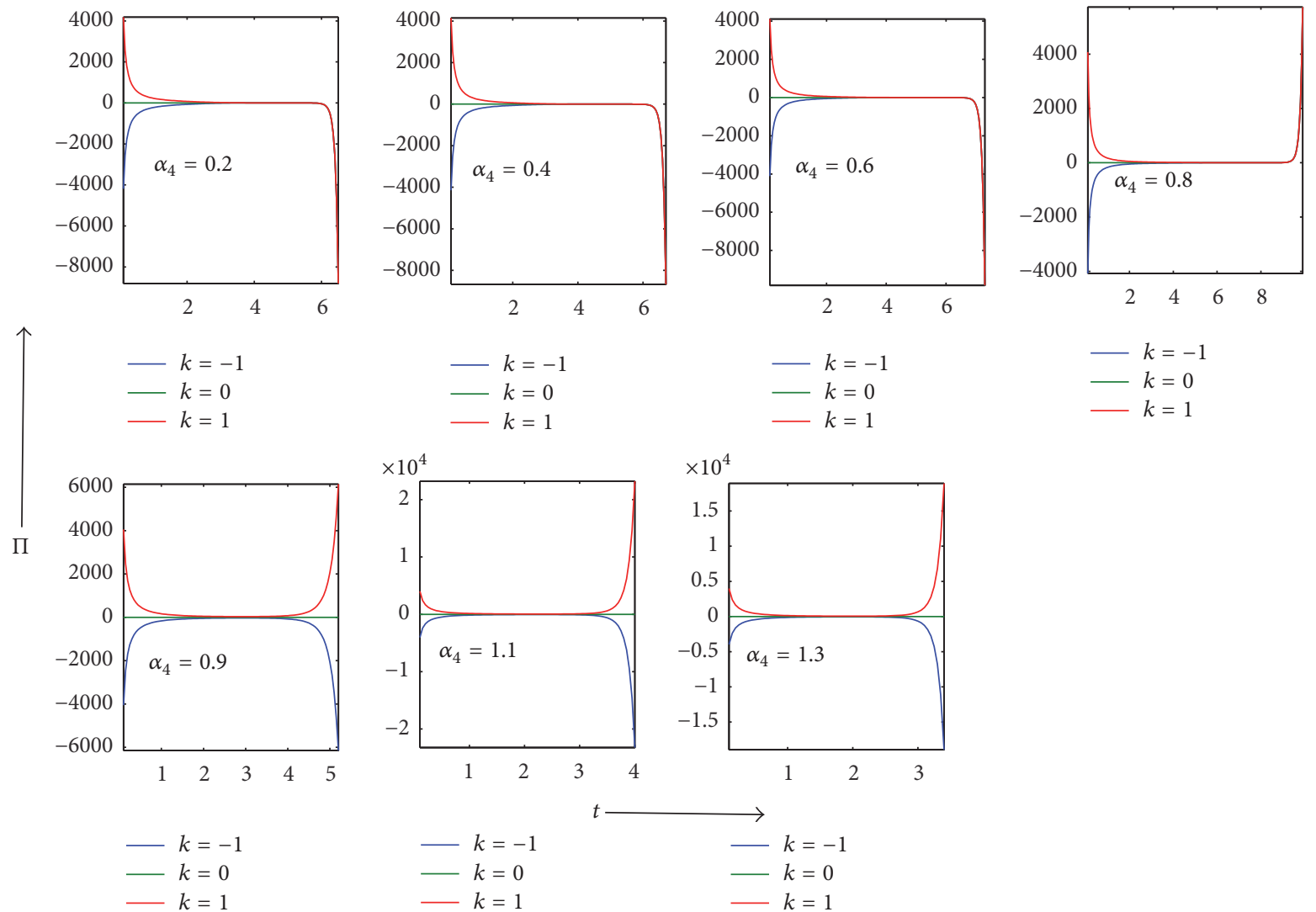

$k=1$

$k=0$
$k=1$

(a)
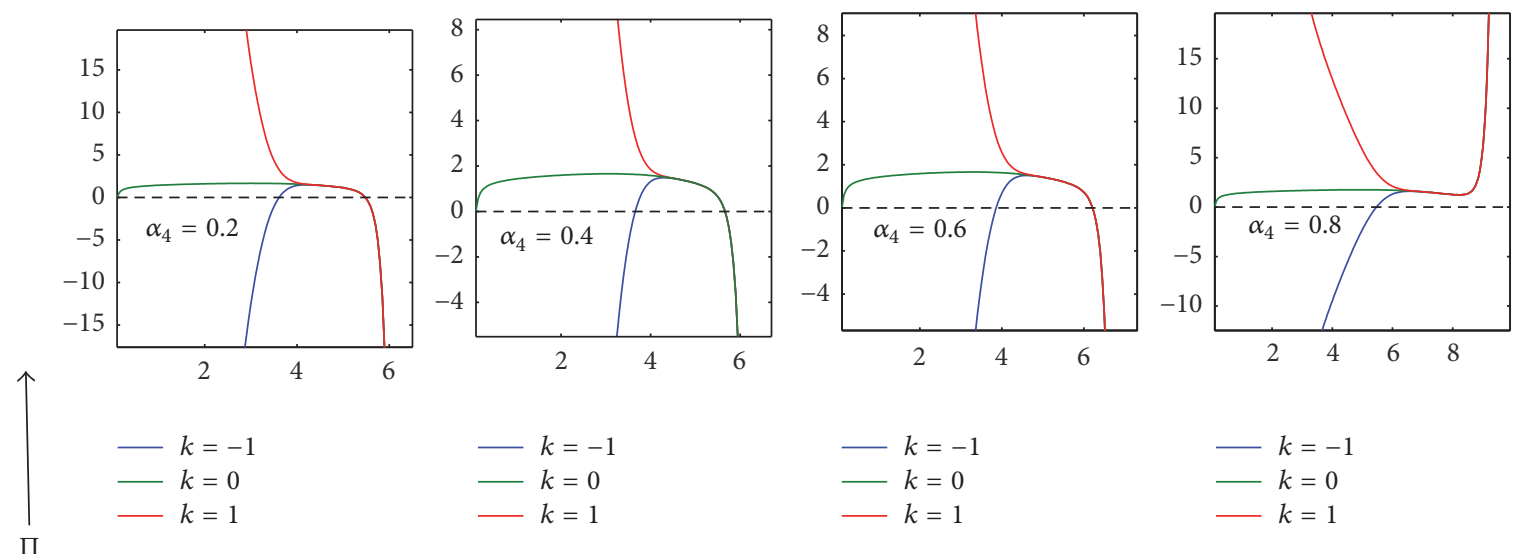
$-k=-1$
$-k=0$
$k=1$
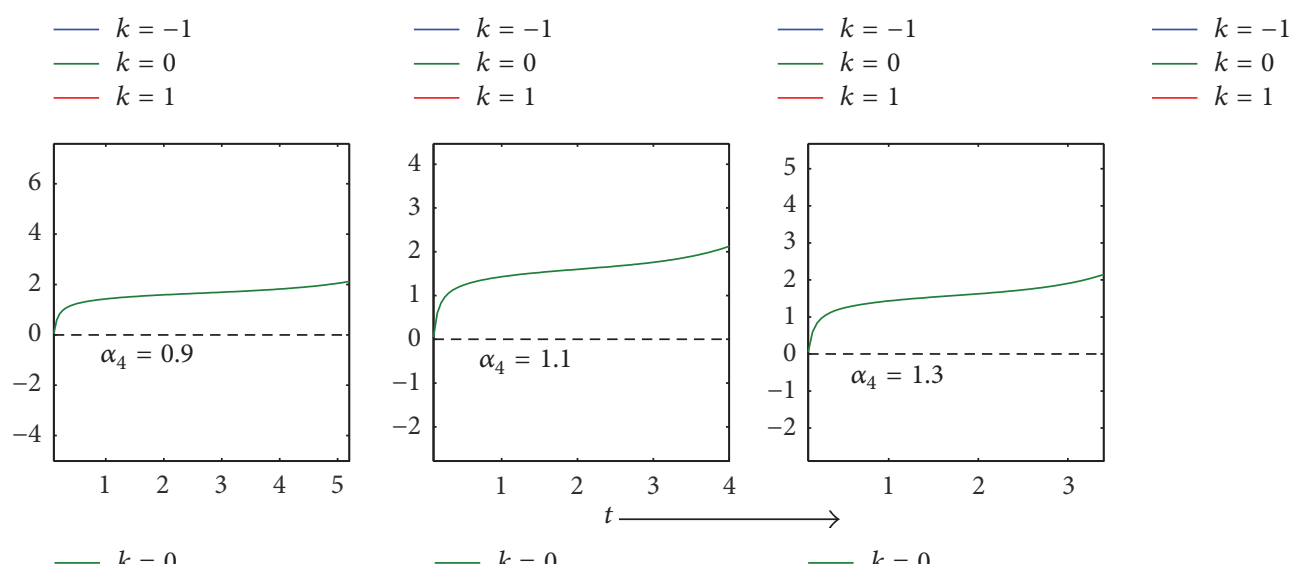

$k=0$

$k=0$

$-k=0$

(b)

Figure 7: Variation of bulk viscous stress $\Pi$ against time for $\alpha_{1}=0.5, \alpha_{2}=0.5, A=B=1, n=0.1, \omega=1, \phi_{0}=1, c_{2}=0.1$, and different $\alpha_{4}$. (b) shows the zooming of (a). 

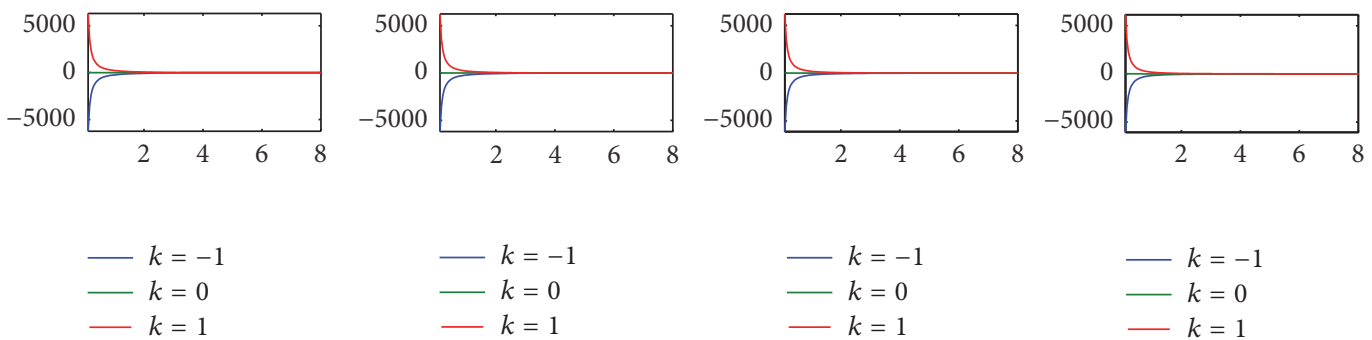

$$
\begin{aligned}
k & =-1 \\
k & =0 \\
k & =1
\end{aligned}
$$

- $k=-1$

$-k=0$

$-k=1$
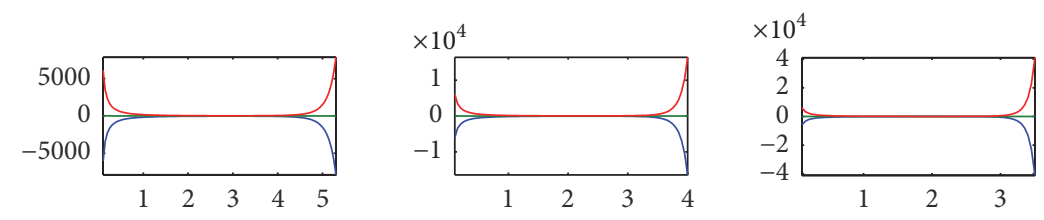

$-k=1$

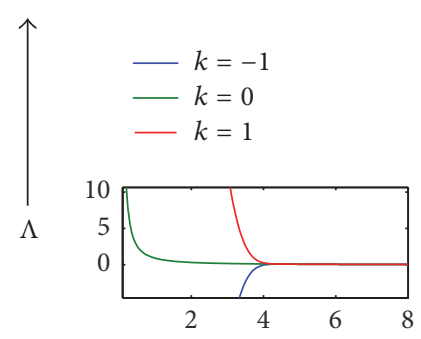

$$
\begin{aligned}
k & =-1 \\
k & =0 \\
k & =1
\end{aligned}
$$

$$
\begin{aligned}
k & =-1 \\
k & =0 \\
k & =1
\end{aligned}
$$
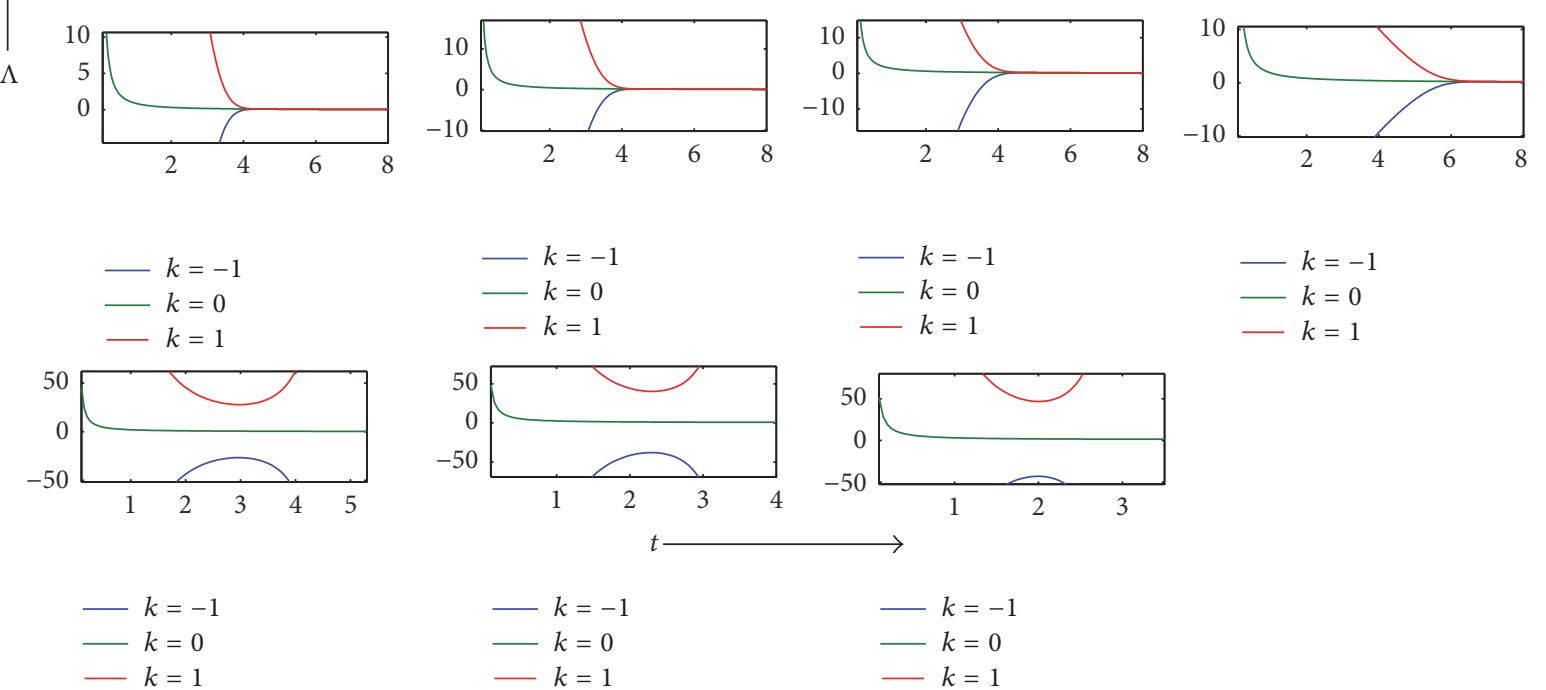
$k=-1$
$-k=0$
$k=1$

$k=-1$

$k=0$

$k=1$

$-k=0$

$\begin{array}{rlrl}k & =-1 \\ k & =0 \\ k & =1 & -k=-1 \\ -k & =0 \\ -k & =1\end{array}$

FIgure 8: Variation of cosmological constant $\Lambda$ against time for $\alpha_{1}=0.5, \alpha_{2}=0.5$, $\omega=1$, $c_{2}=0.1$, and different $\alpha_{4}(0.2,0.4,0.6,0.8,0.9,1.1,1.3)$. Lower two rows represent the magnified portion of the above two rows.

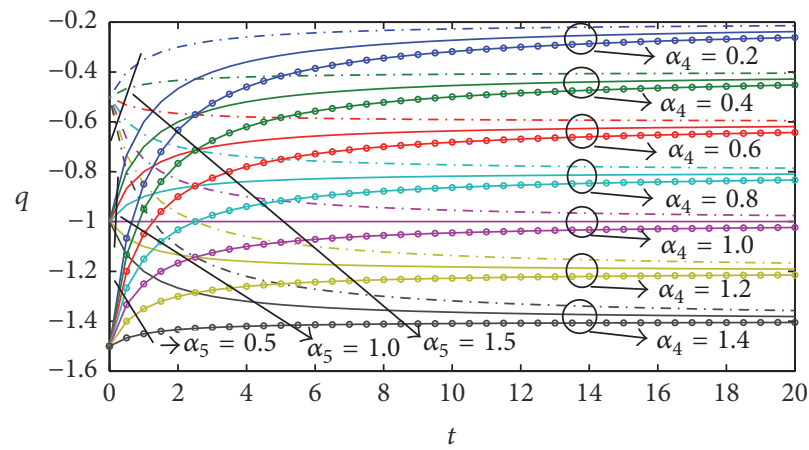

FIGURE 9: Variation of deceleration parameter against time for fixed $\alpha_{5}$ and different $\alpha_{4}$ 


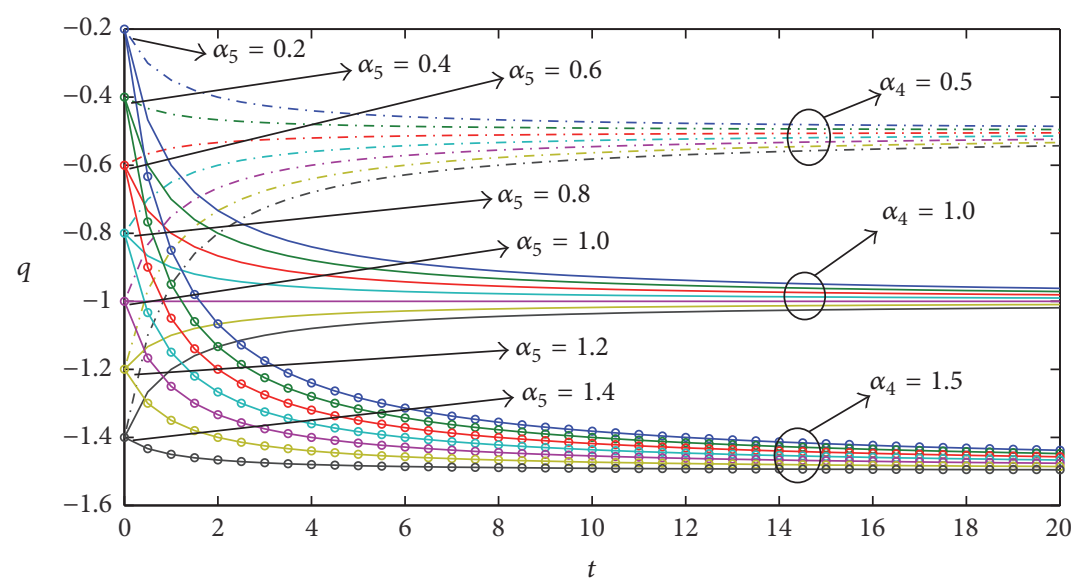

FIgURE 10: Variation of deceleration parameter against time for fixed $\alpha_{4}$ and different $\alpha_{5}$.

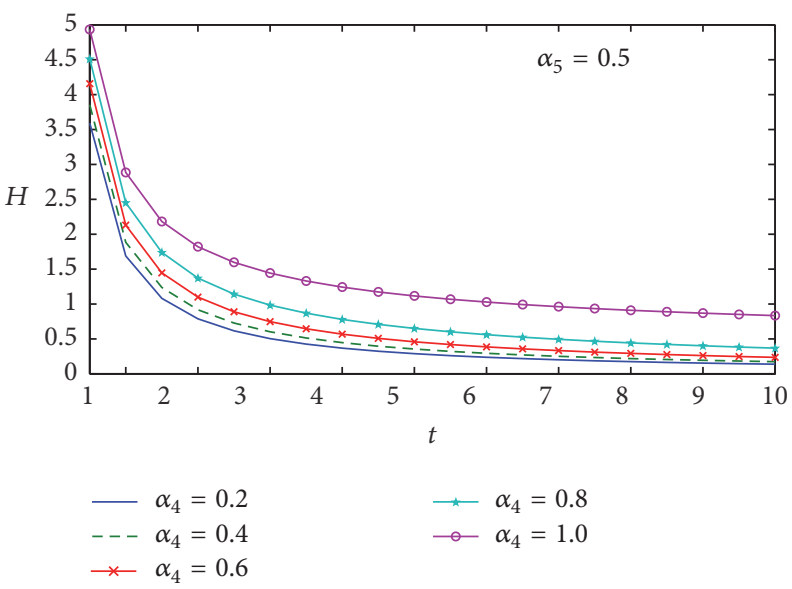

FIGURE 11: Variation of Hubble parameter against time for fixed $\alpha_{5}=0.5$ and different $\alpha_{4}$.

factor $R$ is different for different interval of $\alpha_{4}$ and $\alpha_{5}$. As a representative case, we choose $\alpha_{5}=0.3,0.5$ and different $\alpha_{4}$ and all other parameters as in Figures 12 and 13. In the interval $0<\alpha_{4} \leq 0.2 \& \alpha_{4}>0.9$ and $\alpha_{5}=0.3$, scale factor $R$ increases after taking a bounce where as in $0.3 \leq \alpha_{4} \leq 0.9$ and $\alpha_{5}=0.3$, it increases gradually with the evolution of time (see Figure 12). Similar qualitative behaviour is noticed for $\alpha_{5}=0.5$ and different $\alpha_{4}$ (see Figure 13).

The variation of energy density and pressure against time is presented for model II in Figures 14 and 15, respectively. As a representative case we choose $\alpha_{5}=0.3$ and different $\alpha_{4}$ and all other parameters are as in Figures 14 and 15. The observations are as follows:

(i) Energy density gradually decreases and approaches towards zero with the evolution of time for $0<\alpha_{4} \leq$ 0.3 and $\alpha_{5}=0.3$.

(ii) Energy density is gradually decreased for small interval of time and tends towards infinity with the evolution of time for $0.4 \leq \alpha_{4} \leq 0.9$ and $\alpha_{5}=0.3$. (iii) For $\alpha_{4} \geq 1$ and $\alpha_{5}=0.3$, energy density tends towards zero with time. Here we pointed out that, with the increment of $\alpha_{4}$, the bounce of the energy density increases and gradually tends to zero (see Figure 14).

(iv) Pressure is negative in $0<\alpha_{4} \leq 0.3$ and $1 \leq \alpha_{4} \leq 1.5$ with $\alpha_{5}=0.3$.

(v) Pressure is negative for a small interval of time and gradually increases with time and it takes values from positive to negative in the intervals $0.4 \leq \alpha_{4} \leq 0.9$ and $\alpha_{4} \geq 1.6$ with $\alpha_{5}=0.3$, respectively (see Figure 15 ).

The variation of bulk viscous stress $\Pi$ and cosmological constant $\Lambda$ against time for model II is presented in Figures 16 and 17, respectively. The observations are as follows.

\section{Bulk Viscous Stress $\Pi$ (see Figure 16)}

(i) It is positive valued for flat and closed Universe whereas it is negative valued for open Universe in $0<\alpha_{4} \leq 0.2$ and $1 \leq \alpha_{4} \leq 2$ and $\alpha_{5}=0.3$. 

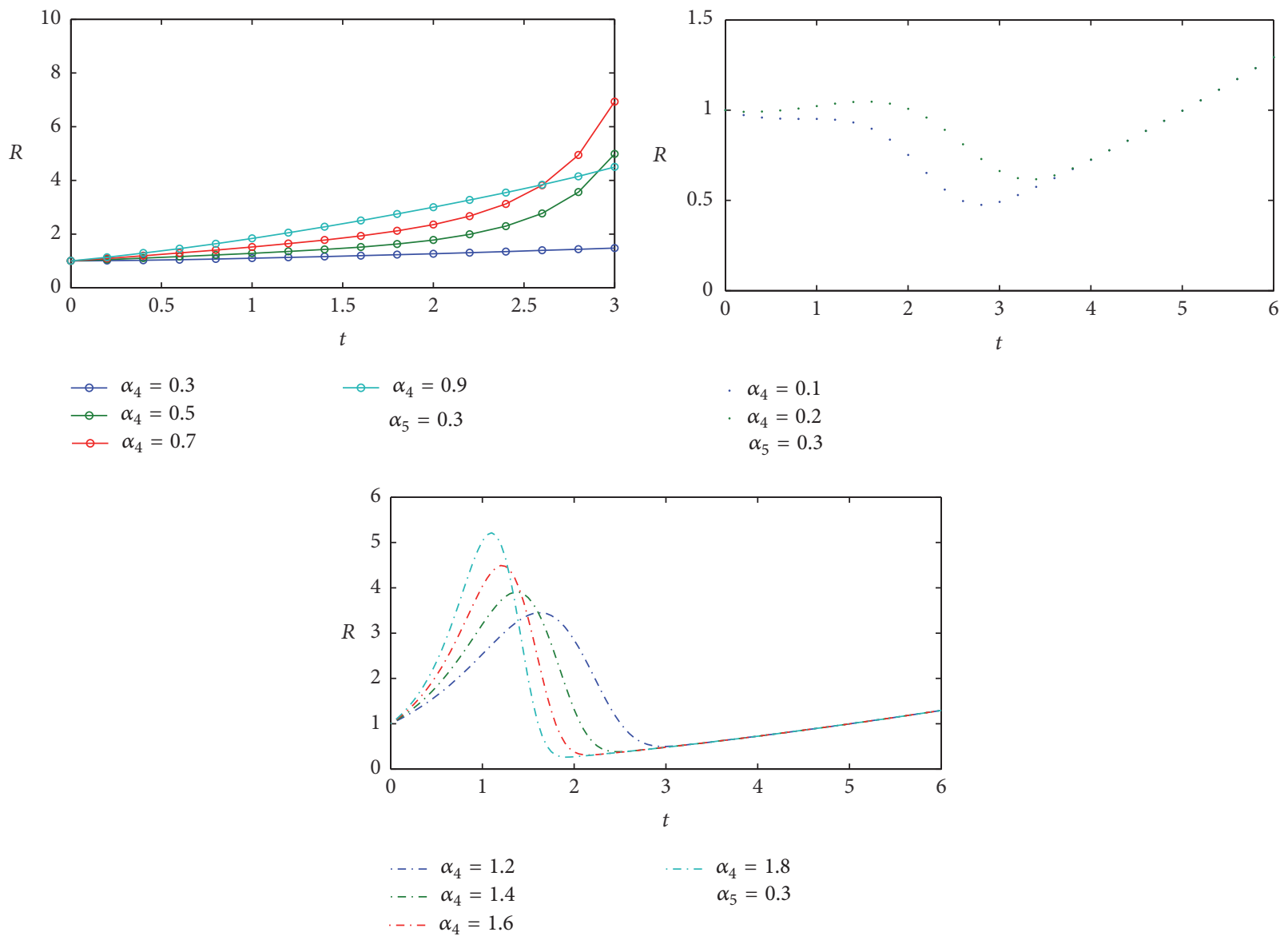

FIGURE 12: Variation of scale factor against time for fixed $\alpha_{5}=0.3$ and different $\alpha_{4}$.

(ii) It is positive-negative valued for flat and closed Universe whereas it is negative-positive-negative valued for open Universe in $0.3 \leq \alpha_{4} \leq 0.8$ and $\alpha_{5}=0.3$.

(iii) It is positive valued for flat and closed Universe whereas it is negative-positive valued for open Universe in $0.8<\alpha_{4} \leq 1$ and $\alpha_{5}=0.3$. Also it approaches towards infinity with the evolution of time in the specified interval of $\alpha_{4}$.

(iv) $\Pi$ is positive valued for flat and closed Universe whereas it is negative-positive-negative valued for open Universe in $\alpha_{4}>2$ and $\alpha_{5}=0.3$.

\section{Cosmological Constant $\Lambda$ (see Figure 17)}

(i) For $0<\alpha_{4}<0.3$ and $\alpha_{5}=0.3, \Lambda$ is positive valued for flat and closed Universe whereas it is negative value for open Universe. In case of flat Universe $\Lambda \rightarrow 0$ when $t \rightarrow \infty$ but for open and closed Universe $\Lambda \rightarrow$ $\infty$ and $\Lambda \rightarrow-\infty$ with time respectively.

(ii) For $0.3<\alpha_{4} \leq 0.9$ and $\alpha_{5}=0.3, \Lambda \rightarrow 0$ when $t \rightarrow \infty$ for open, flat, and closed Universe. In case of flat and closed Universe, cosmological constant is positive valued whereas in open Universe it is negative-positive valued. (iii) It is positive valued for flat and closed Universe but it is negative-positive-negative valued for open Universe in $\alpha_{4}>0.9$ and $\alpha_{5}=0.3$.

3.3. Model III. The deceleration parameter $q$ in (6) for $\alpha_{2}<0$ and $\alpha_{3}=0$ takes the form

$$
q=-\frac{\alpha_{5}}{1+t}, \quad \alpha_{2}=-\alpha_{5}, \alpha_{5}>0
$$

Here we noticed that $q<0$ for $\alpha_{5}>0$, which means that our Universe is accelerating with the evolution of time.

For model III, the physical parameters are obtained as follows:

The Hubble parameter in (12) takes the form

$$
H=\frac{1}{t-\alpha_{5} \ln (1+t)}
$$

The scale factor $R(t)$ in (16) is expressed as

$$
R=c_{2} t^{1 /\left(1-\alpha_{5}\right)} e^{T_{1}(t)}
$$



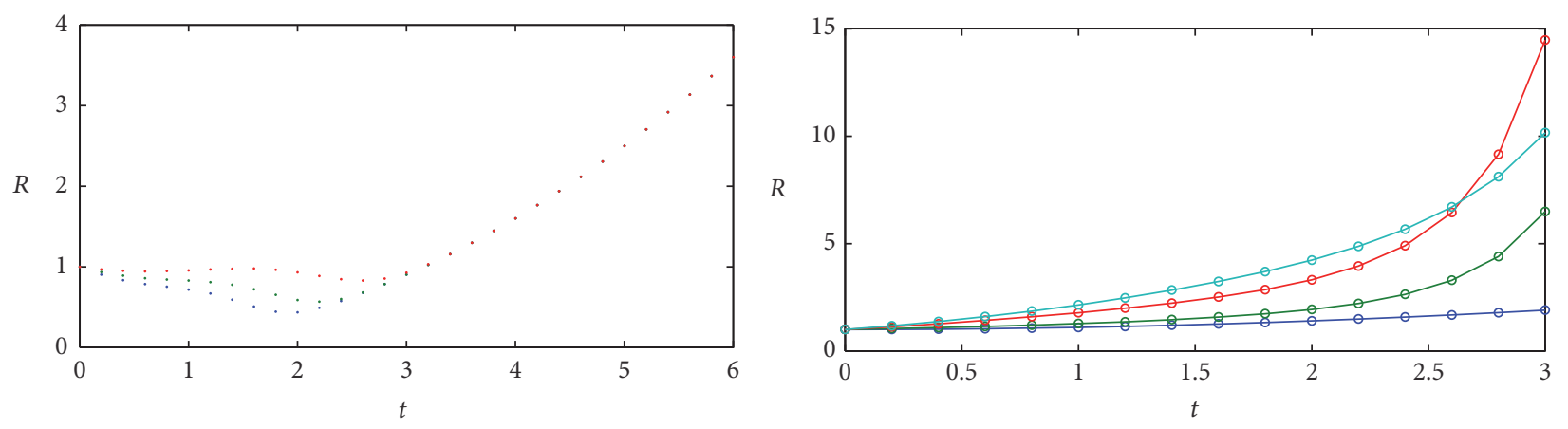
$\alpha_{4}=0.2$
$\alpha_{5}=0.5$
$\begin{aligned} \multimap & \alpha_{4}=0.5 \\ \rightarrow & \alpha_{4}=0.6\end{aligned}$
$\therefore \alpha_{4}=0.9$
$\alpha_{4}=0.3$
$\begin{array}{ll}\multimap & \alpha_{4}=0.6 \\ \rightarrow & \alpha_{4}=0.8\end{array}$
$\alpha_{5}=0.5$
$\alpha_{4}=0.4$

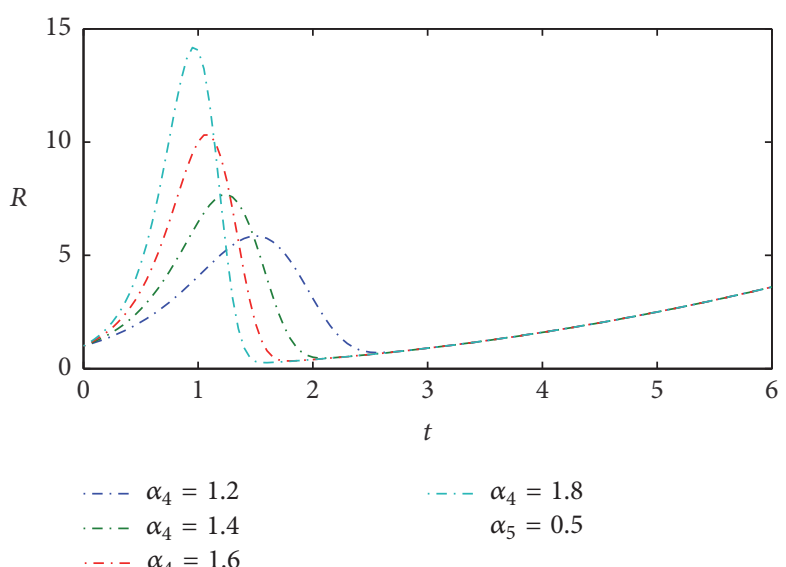

FIGURE 13: Variation of scale factor against time for fixed $\alpha_{5}=0.5$ and different $\alpha_{4}$.

where $T_{1}(t)=k_{0} t+k_{1}\left(t^{2} / 2\right)+k_{2}\left(t^{3} / 3\right)+k_{3}\left(t^{4} / 4\right)+k_{4}\left(t^{5} / 5\right)+$ $O\left(t^{6}\right)$. And

$$
\begin{aligned}
k_{0} & =-\frac{\alpha_{5}}{2\left(1-\alpha_{5}\right)^{2}} \\
k_{1} & =\frac{1}{1-\alpha_{5}}\left[\frac{\alpha_{5}^{2}}{4\left(1-\alpha_{5}\right)^{2}}+\frac{\alpha_{5}}{3\left(1-\alpha_{5}\right)}\right] \\
k_{2} & =-\frac{1}{1-\alpha_{5}}\left[\frac{\alpha_{5}}{4\left(1-\alpha_{5}\right)}+\frac{\alpha_{5}^{2}}{3\left(1-\alpha_{5}\right)^{2}}\right. \\
& \left.+\frac{\alpha_{5}^{3}}{8\left(1-\alpha_{5}\right)^{3}}\right] \\
k_{3} & =\frac{1}{1-\alpha_{5}}\left[\frac{\alpha_{5}}{5\left(1-\alpha_{5}\right)}+\frac{13 \alpha_{5}^{2}}{36\left(1-\alpha_{5}\right)^{2}}\right. \\
& \left.+\frac{\alpha_{5}^{3}}{4\left(1-\alpha_{5}\right)^{3}}+\frac{\alpha_{5}^{4}}{16\left(1-\alpha_{5}\right)^{4}}\right]
\end{aligned}
$$

$$
\begin{aligned}
k_{4} & =-\frac{1}{1-\alpha_{5}}\left[\frac{\alpha_{5}}{6\left(1-\alpha_{5}\right)}+\frac{11 \alpha_{5}^{2}}{30\left(1-\alpha_{5}\right)^{2}}\right. \\
& \left.+\frac{17 \alpha_{5}^{3}}{48\left(1-\alpha_{5}\right)^{3}}+\frac{\alpha_{5}^{4}}{6\left(1-\alpha_{5}\right)^{4}}\right] .
\end{aligned}
$$

The FRW space-time metric in (3) takes the form

$$
\begin{aligned}
d s^{2} & =d t^{2}-c_{2}^{2} t^{2 /\left(1-\alpha_{5}\right)} e^{2 T_{1}(t)}\left[\frac{d r^{2}}{1-k r^{2}}\right. \\
& \left.+r^{2}\left(d \theta^{2}+\sin ^{2} \theta d \phi^{2}\right)\right]
\end{aligned}
$$

with the above mentation $k_{i},(i=0,1,2,3,4)$. The energy density $(\rho)$, pressure $(p)$, bulk viscous stress $(\Pi)$, and cosmological constant $(\Lambda)$ in (17), (18), (19), and (20) takes the form

$$
\rho=\frac{k_{5} t^{\alpha_{1} /\left(1-\alpha_{5}\right)} e^{\alpha_{1} T_{1}(t)}\left(\rho_{1}+\rho_{2} t\right)}{(1+t)\left[t-\alpha_{5} \ln (1+t)\right]^{2}},
$$

where $k_{5}=\phi_{0} c_{2}^{\alpha_{1}} / 8 \pi, \rho_{1}=\left(3+2 w+w \alpha_{5}\right) \alpha_{1}-3 \alpha_{5}, \rho_{2}=$ $(3+2 w) \alpha_{1}$. 

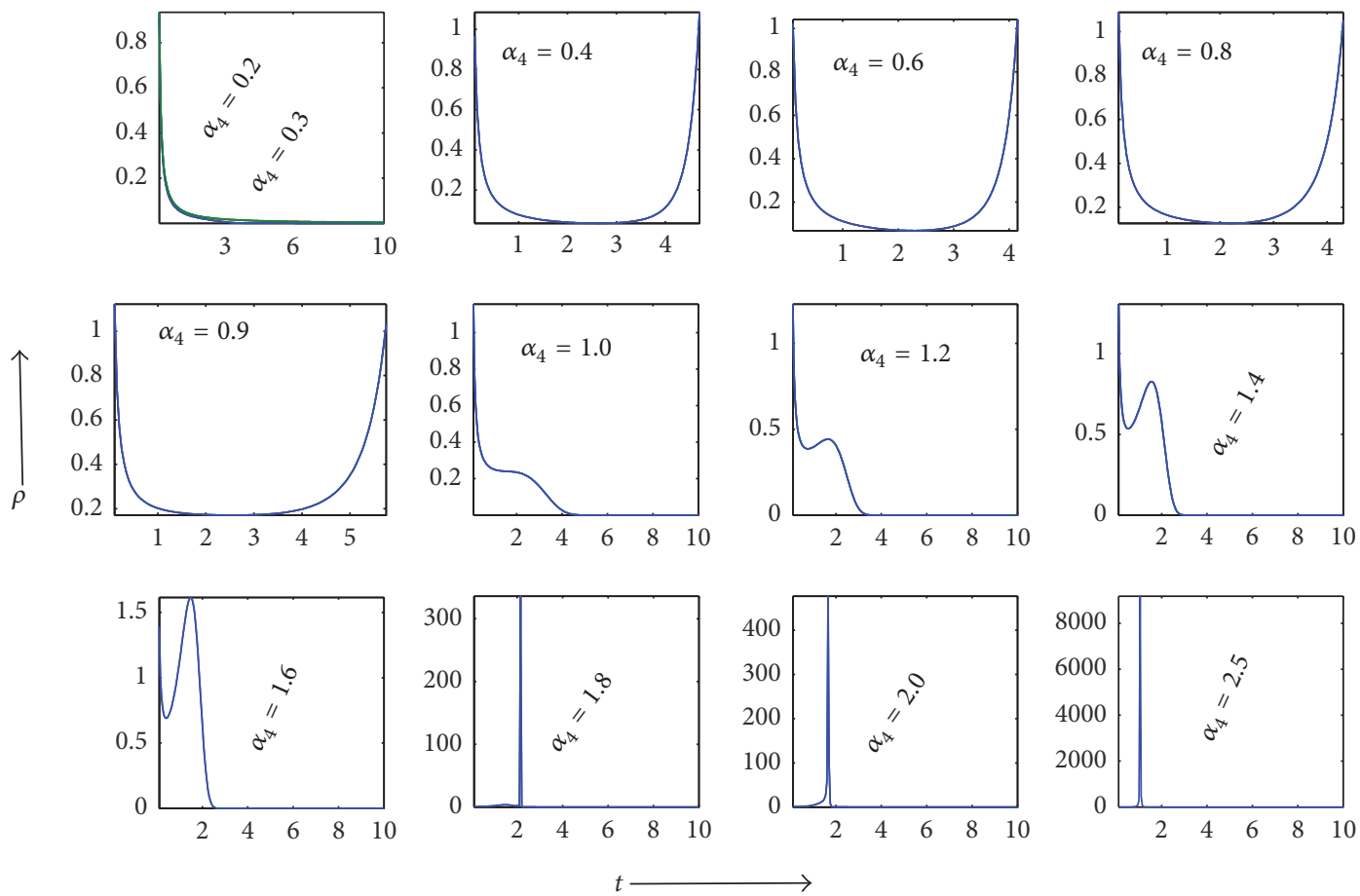

(a)
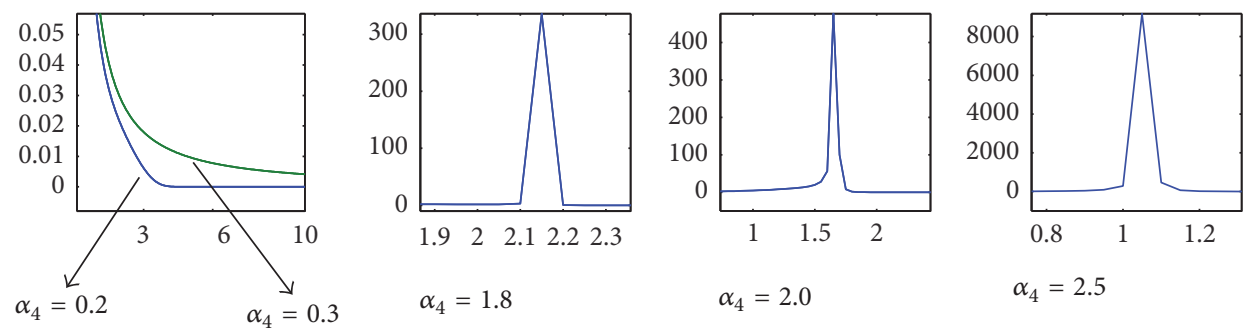

$\alpha_{4}=2.0$

$\alpha_{4}=2.5$

(b)

FIGURE 14: Variation of energy density $\rho$ against time for $\alpha_{1}=0.5, \alpha_{5}=0.3, \omega=1, \phi_{0}=1, c_{2}=0.1$, and different $\alpha_{4}$. (b) shows the zooming of (a).

$$
\begin{gathered}
p=\frac{A k_{5}^{n+1} t^{(n+1) \alpha_{1} /\left(1-\alpha_{5}\right)} e^{(n+1) \alpha_{1} T_{1}(t)}\left(\rho_{1}+\rho_{2} t\right)^{n+1}-B(1+t)^{n+1}\left[t-\alpha_{5} \ln (1+t)\right]^{2 n+2}}{k_{5}^{n} t^{n \alpha_{1} /\left(1-\alpha_{5}\right)} e^{n \alpha_{1} T_{1}(t)}\left(\rho_{1}+\rho_{2} t\right)^{n}(1+t)\left[t-\alpha_{5} \ln (1+t)\right]^{2}} \\
\left.k_{5}^{n+1} t^{(n+1) \alpha_{1} /\left(1-\alpha_{5}\right)} e^{(n+1) \alpha_{1} T_{1}(t)}\left[\frac{\rho_{1}+\rho_{2} t}{(1+t)\left[t-\alpha_{5} \ln (1+t)\right]^{2}}\right]^{2}+2 k / c_{2}^{2} t^{2 /\left(1-\alpha_{5}\right)} e^{2 T_{1}(t)}\right]+B \\
\times \Pi=\frac{\left.\left[\left(\Pi_{1}+\Pi_{2} t\right) /(1+t)\left[t-\alpha_{5} \ln (1+t)\right]^{2}+\alpha_{5}^{n} \ln (1+t)\right]^{2}\right]^{n}}{k_{5}^{n} t^{n \alpha_{1} /\left(1-\alpha_{5}\right)} e^{n \alpha_{1} T_{1}(t)}\left[\left(\rho_{1}+\rho_{2} t\right) /(1+t)[t-\alpha \ln \right.}
\end{gathered}
$$

where $\Pi_{1}=-(1+w) \alpha_{1}^{2}+(1+3 A) \alpha_{5}-\left[\left(\alpha_{5}+2\right)(1+A) w+1+3 A+\right.$ $\left.\alpha_{5}\right] \alpha_{1}+2$ and $\Pi_{2}=-(1+w) \alpha_{1}^{2}-\left[\left(\alpha_{5}+2\right)(1+A) w+1+3 A\right] \alpha_{1}+2$.

$$
\Lambda=\frac{\Lambda_{1}+\Lambda_{2} t}{(1+t)\left[t-\alpha_{5} \ln (1+t)\right]^{2}}+\frac{3 k}{c_{2}^{2} t^{2 /\left(1-\alpha_{5}\right)} e^{2 T_{1}(t)}}
$$



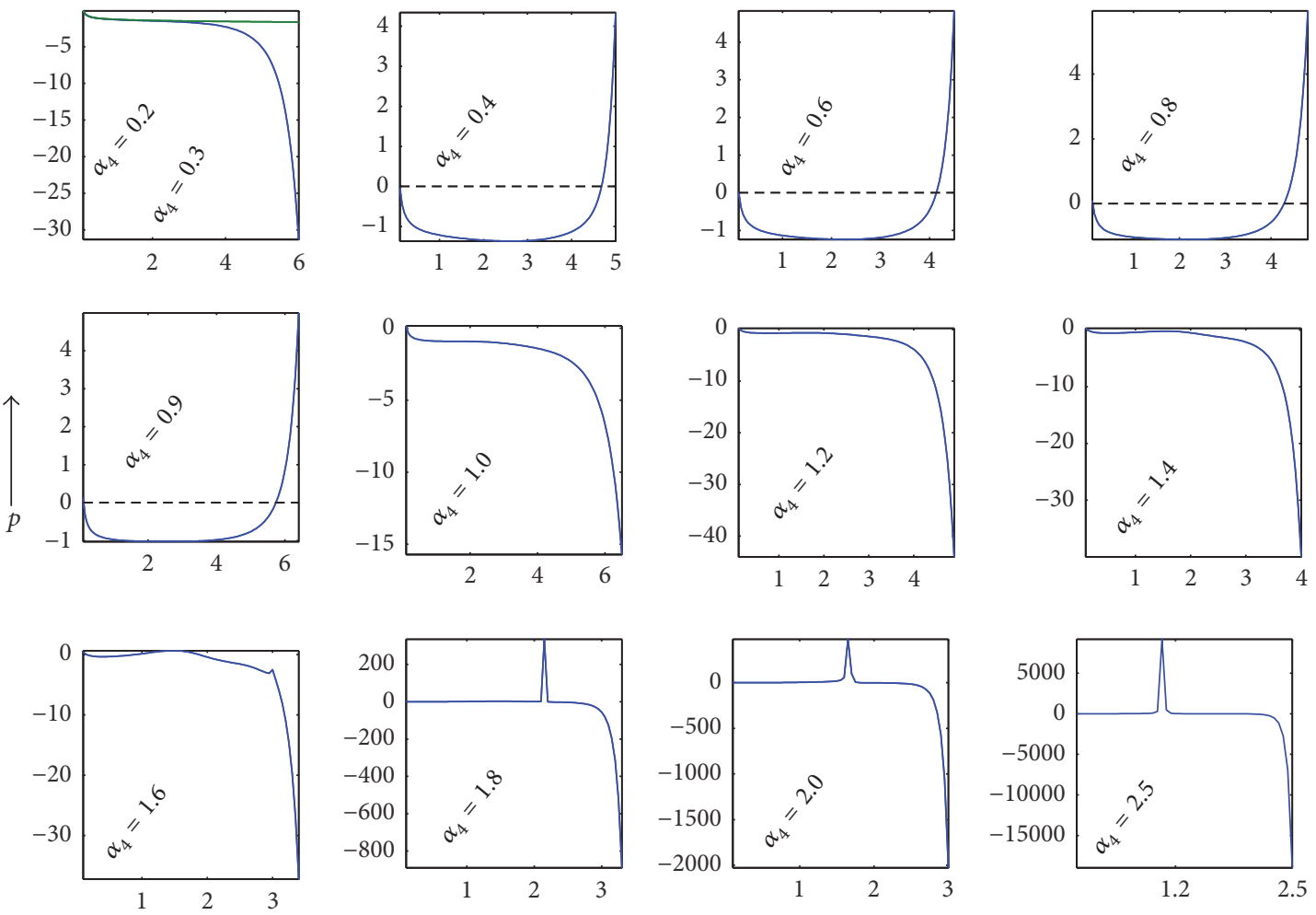

(a)
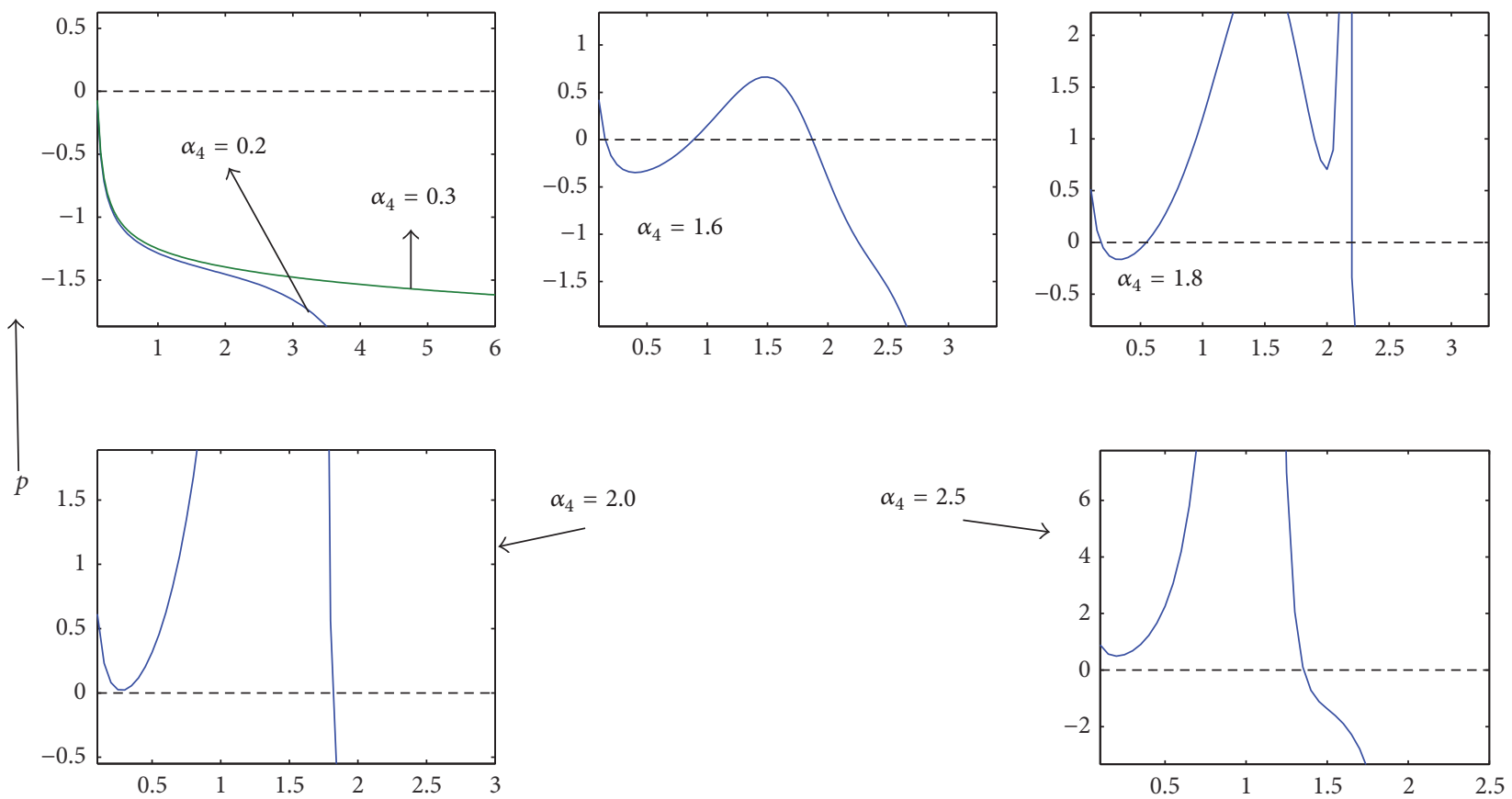

(b)

FiguRE 15: Variation of pressure $p$ against time for $\alpha_{1}=0.5, \alpha_{5}=0.3, A=B=1, n=0.1, \omega=1, \phi_{0}=1, c_{2}=0.1$, and different $\alpha_{4}$. (b) shows the zooming of (a). 

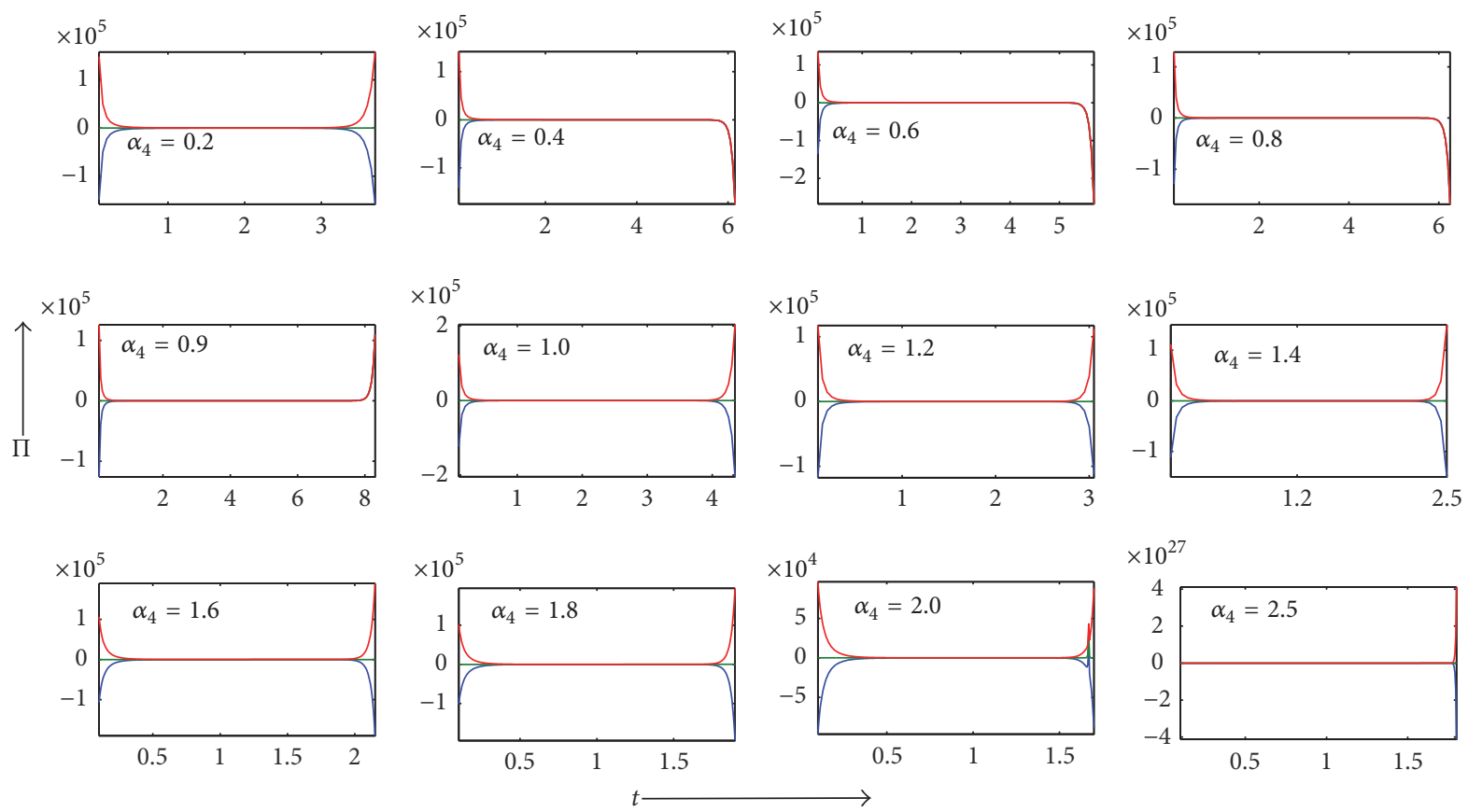

(a)
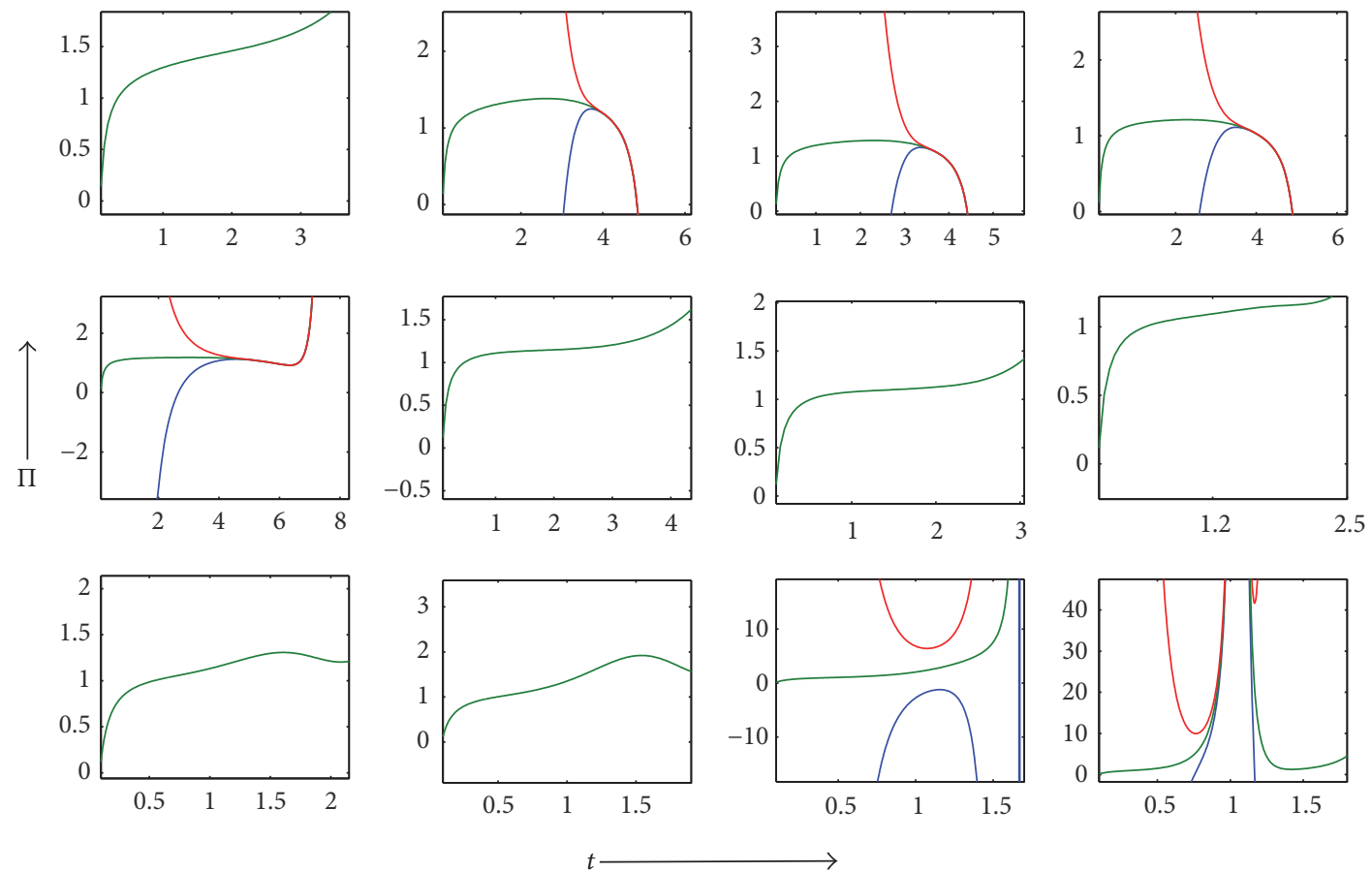

(b)

FIGURE 16: Variation of bulk viscous stress $\Pi$ against time for $\alpha_{1}=0.5, \alpha_{5}=0.3, A=B=1, n=0.1, \omega=1, \phi_{0}=1, c_{2}=0.1$, and different $\alpha_{4}$. (b) shows the zooming of (a). Blue line, Green line, and Red line represent $k=-1, k=0$, and $k=1$, respectively. 

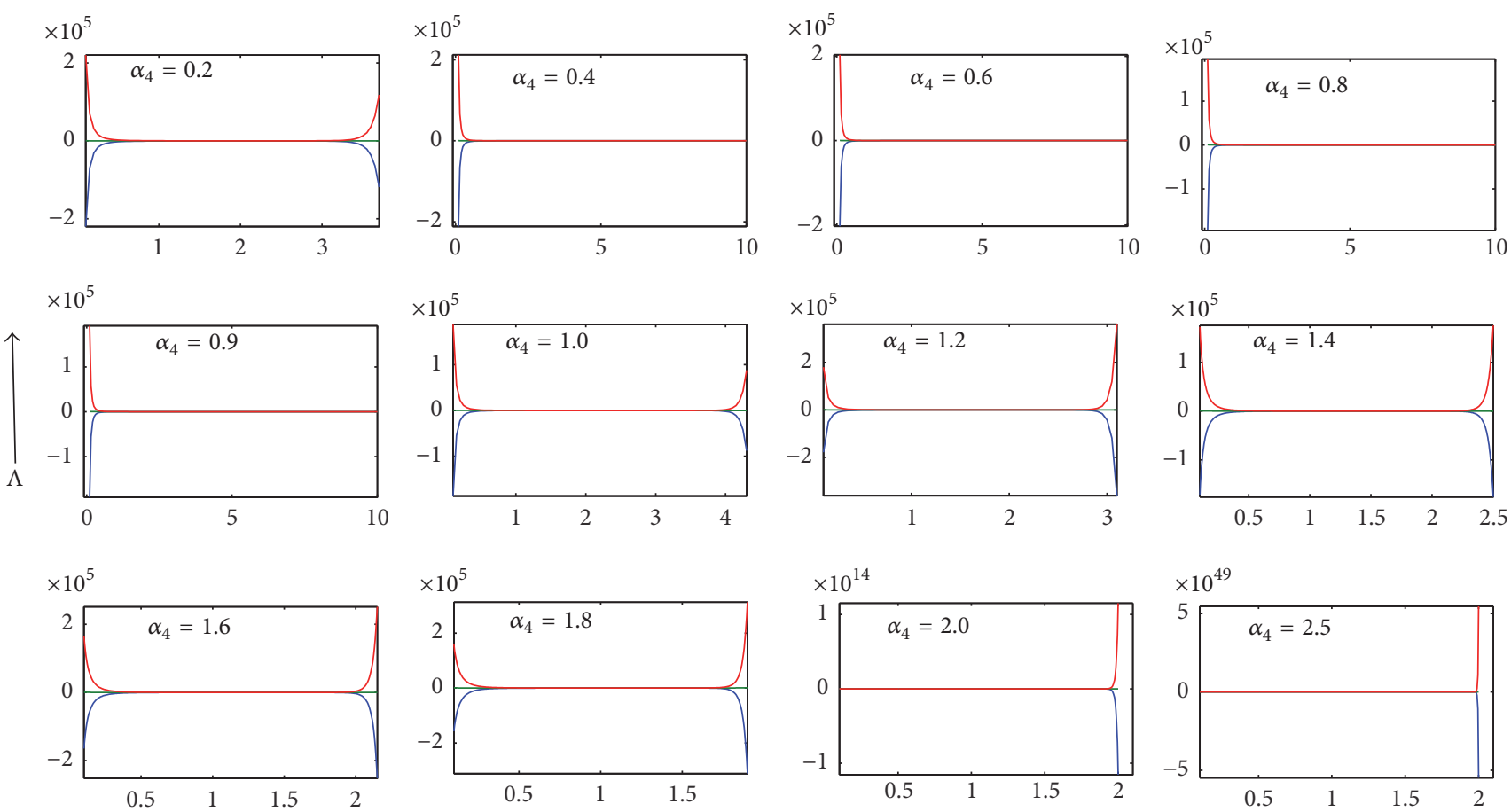

(a)
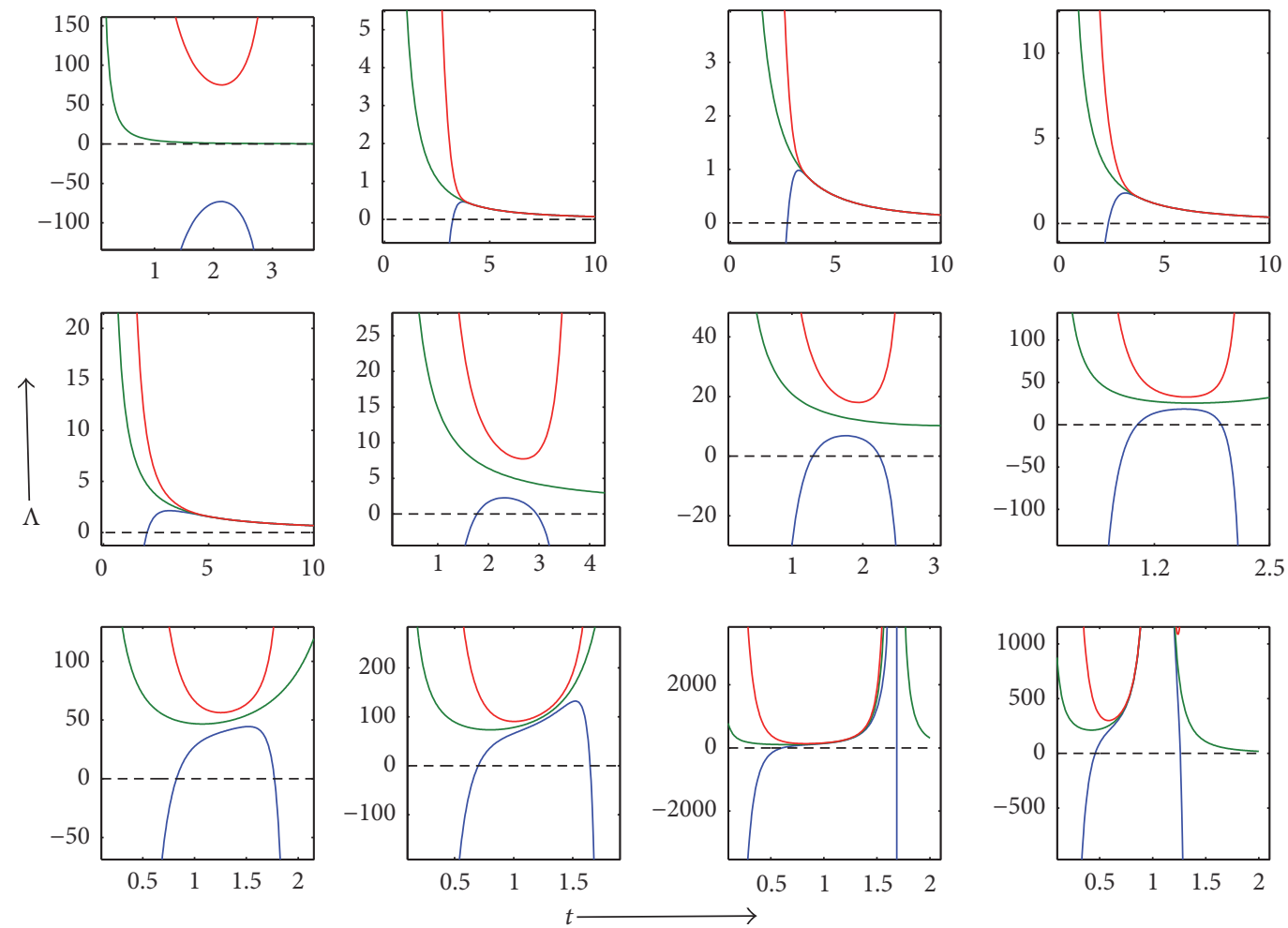

(b)

FIGURE 17: Variation of cosmological constant $\Lambda$ against time for $\alpha_{1}=0.5, \alpha_{5}=0.3, \omega=1, c_{2}=0.1$, and different $\alpha_{4}$. (b) shows the zooming of (a). Blue line, Green line, and Red line represent $k=-1, k=0$, and $k=1$, respectively. 


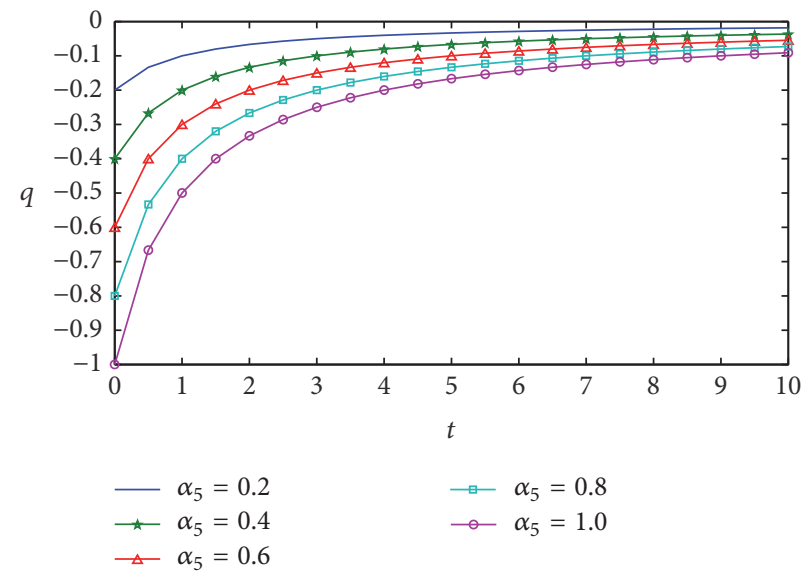

FigURE 18: Variation of deceleration parameter against time for different $\alpha_{5}$.

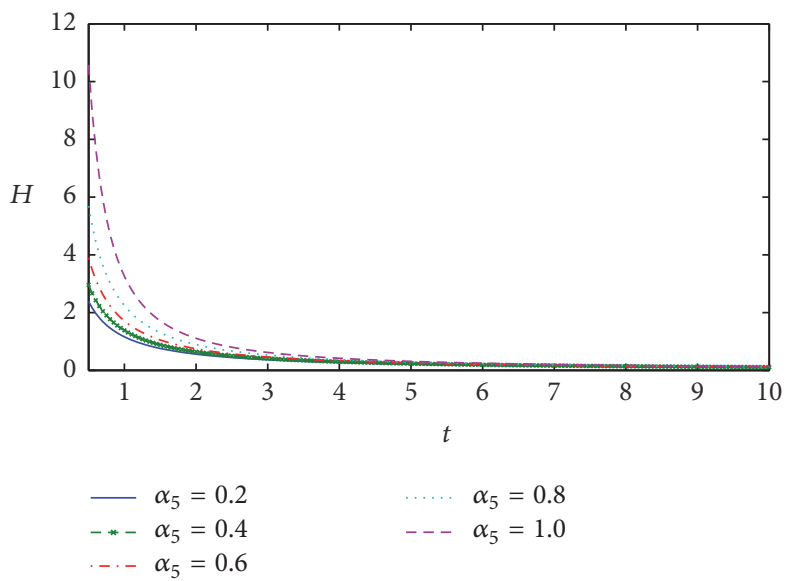

FIGURE 19: Variation of Hubble parameter against time different $\alpha_{5}$.

where $\Lambda_{1}=-\left[0.5 w \alpha_{1}^{2}+w\left(\alpha_{5}+2\right) \alpha_{1}-3\left(\alpha_{5}+1\right)\right]$ and $\Lambda_{2}=$ $-0.5 w \alpha_{1}^{2}-2 w \alpha_{1}+3$.

The profile of deceleration parameter, Hubble parameter, and scale factor against time is plotted in the Figures 18, 19, and 20, respectively, for model III. The observations are as follows:

(i) Deceleration parameter $q$ is a negative valued function of time and approaches towards zero with the evolution of time. In other words we can say, at early time, our Universe is accelerating and follows an expansion with constant rate at late time (see Figure 18).

(ii) Hubble parameter $H$ is a decreasing function of time and $H \rightarrow 0$ when $t \rightarrow \infty$. Also in this case, the higher the value of $\alpha_{5}$, the higher the value of Hubble parameter (see Figure 19).

(iii) Scale factor $R$ is an increasing function of time and $R \rightarrow \infty$ when $t \rightarrow \infty$. Equation (39) indicates that $R$ is not defined for $\alpha_{5}=1$. As a representative case, we considered $0<\alpha_{5}<1$ (see Figure 20).

Figures 21 and 22 depict the energy density and pressure profile against time. respectively. For $\alpha_{5} \geq 1$, energy density possesses physical unrealistic behaviour, so $\alpha_{5}$ is restricted to $0<\alpha_{5}<1$. It is noticed that energy density is a decreasing function of time and $\rho \rightarrow 0$ when $t \rightarrow \infty$ (see Figure 21). Also pressure is a negative quantity with the evolution of time (see Figure 22).

The profile of bulk viscous stress and cosmological constant against time is depicted in Figures 23 and 24, respectively, for model III. Bulk viscous stress is positive valued for flat and closed Universe whereas it is negative valued for open Universe. Similar quantitative behaviour is observed for cosmological constant. In case of flat Universe, cosmological constant is a decreasing function of time and tends to zero with the evolution of time. 

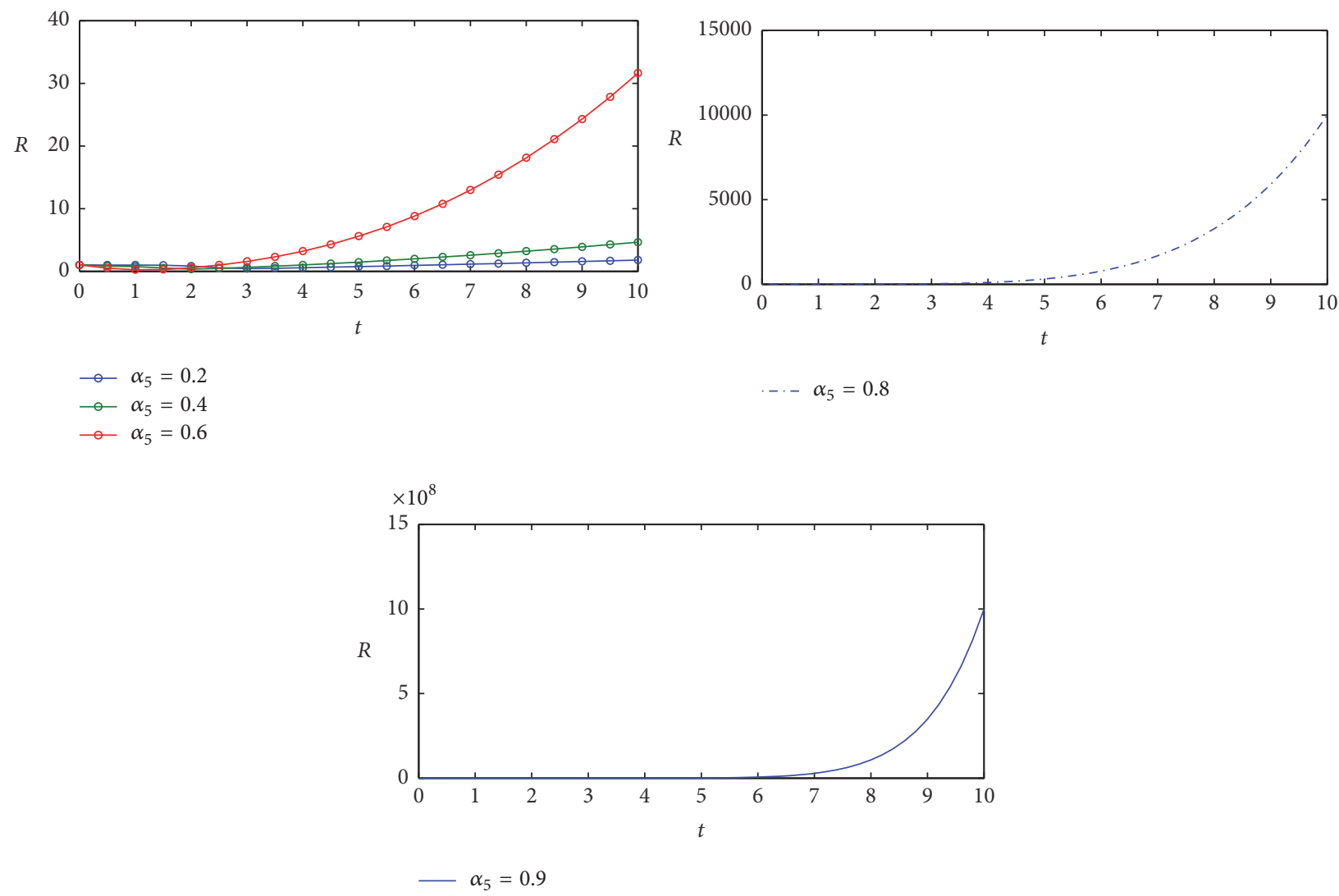

FIgURE 20: Variation of scale factor against time for different $\alpha_{5}$.

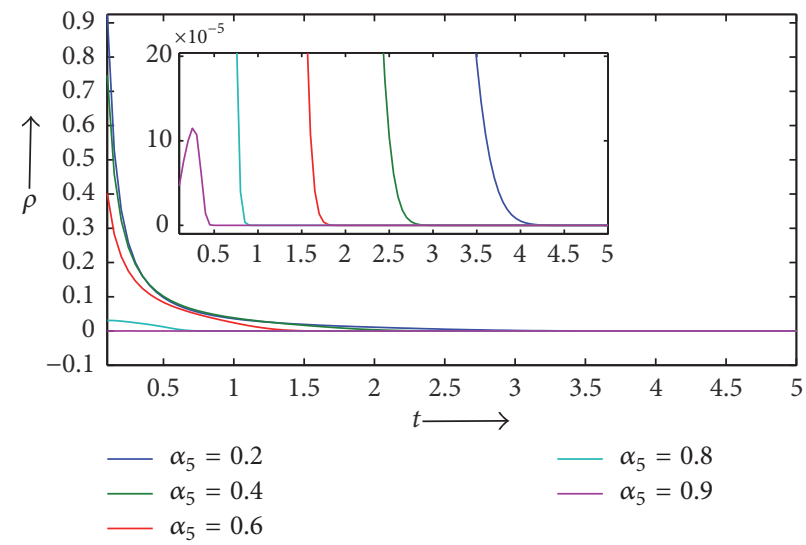

FIgURE 21: Variation of energy density $\rho$ against time for $\alpha_{1}=0.5, \omega=1, \phi_{0}=1, c_{2}=0.1$, and different $\alpha_{5}$.

\section{Final Statements}

In this article, we have studied the FRW cosmological model with modified Chaplygin gas in the framework of BransDicke theory. The approximated exact solution is obtained for modified Einstein's field equation with the help of proposed form of deceleration parameter as in (6). We have presented three different cosmological models based on the choice of $\alpha_{2}$ and $\alpha_{3}$. The physical parameters involved in these three models are physically acceptable for some interval of $\alpha_{2}$ and $\alpha_{3}$, which follow the observational data. Here we would like conclude that, for physically acceptable cosmological models, the choice of $\alpha_{2}$ and $\alpha_{3}$ is crucial.

\section{Conflicts of Interest}

The authors declare that they have no conflicts of interest. 

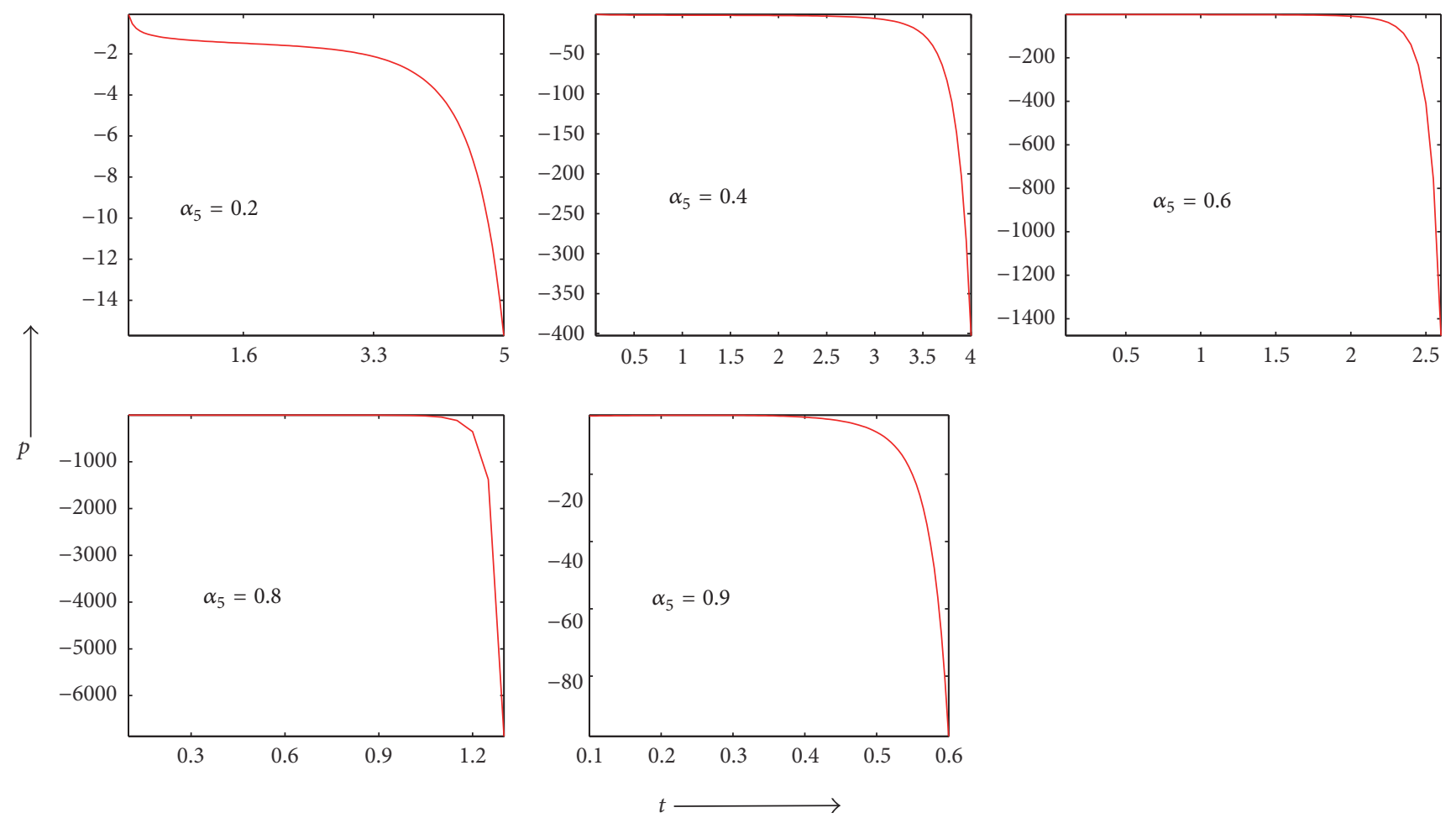

(a)
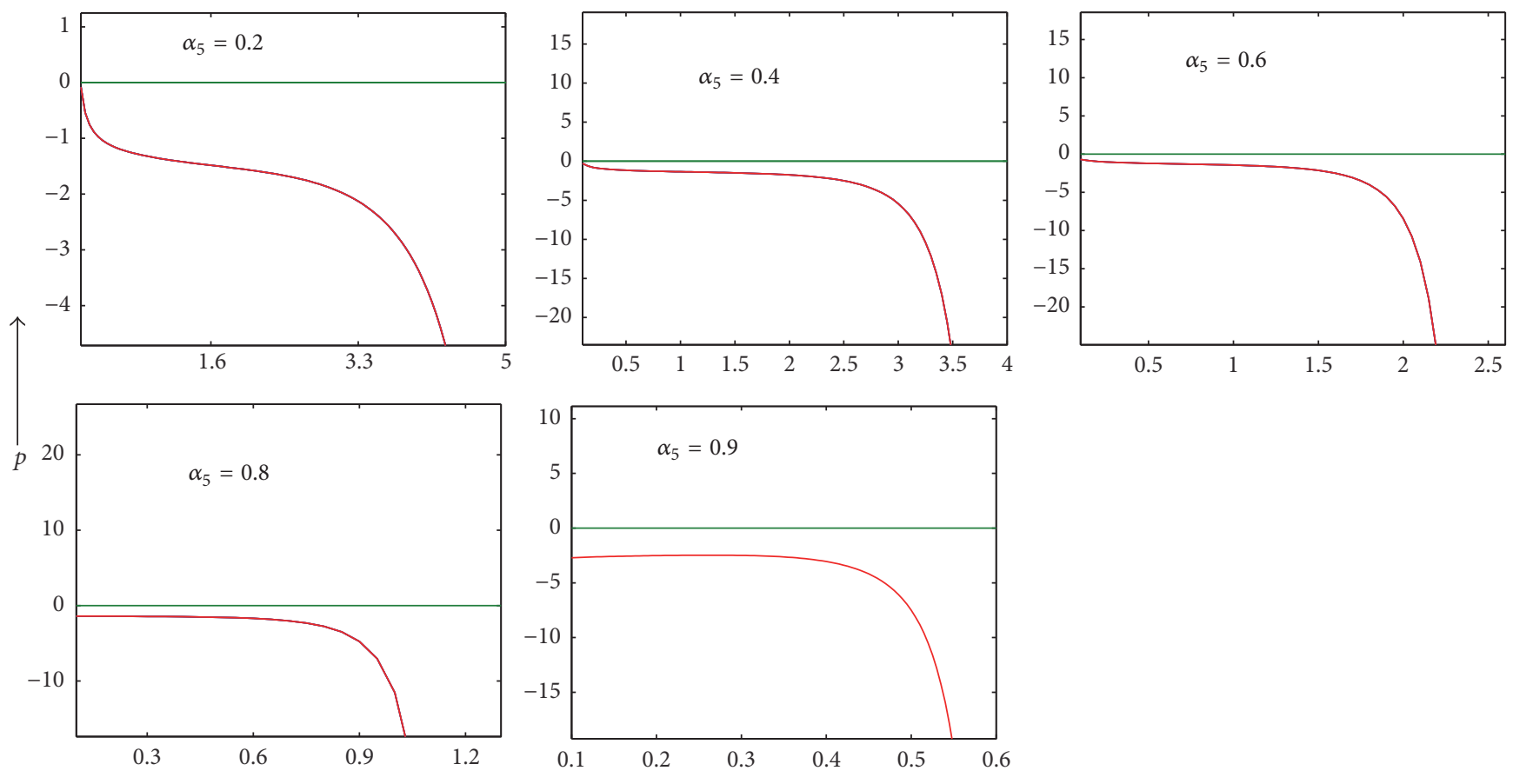

(b)

Figure 22: Variation of pressure $p$ against time for $\alpha_{1}=0.5, A=1, B=1, n=0.1, \omega=1, \phi_{0}=1, c_{2}=0.1$, and different $\alpha_{5}$. (b) shows the zooming of (a). 

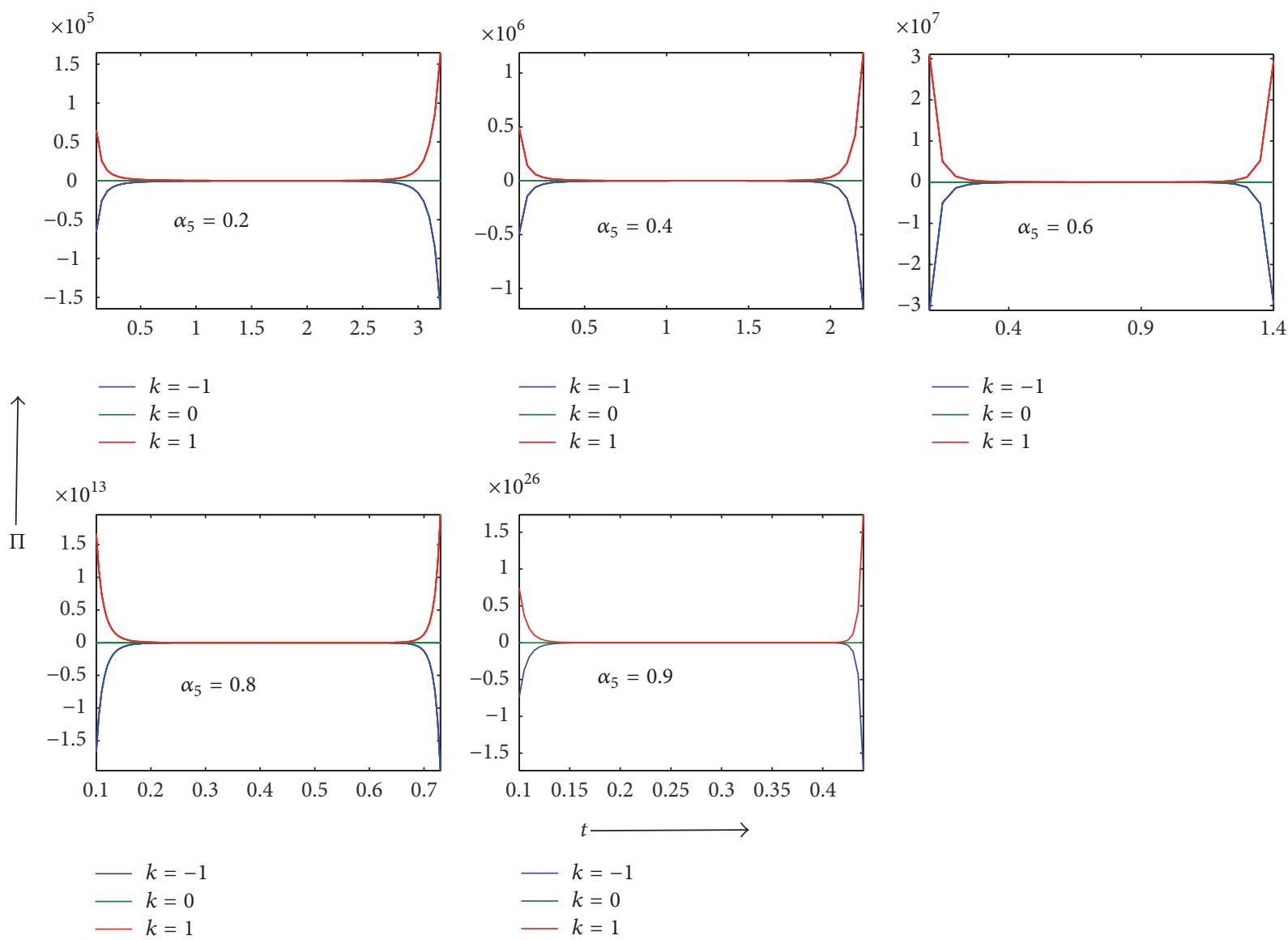
$k=-1$
$-k=0$
$-k=1$

$-k=-1$

$-k=0$

$\times 10^{26}$

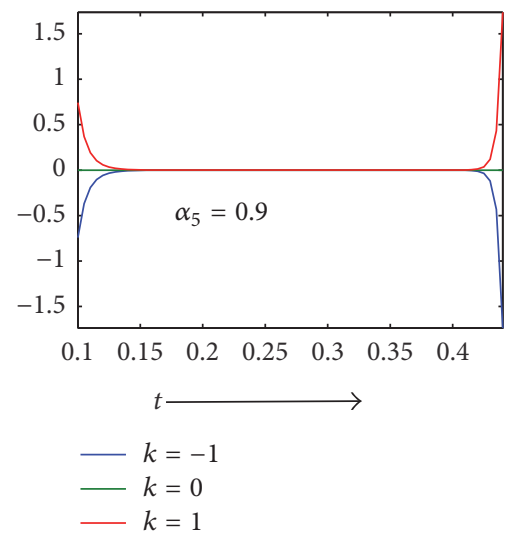

(a)
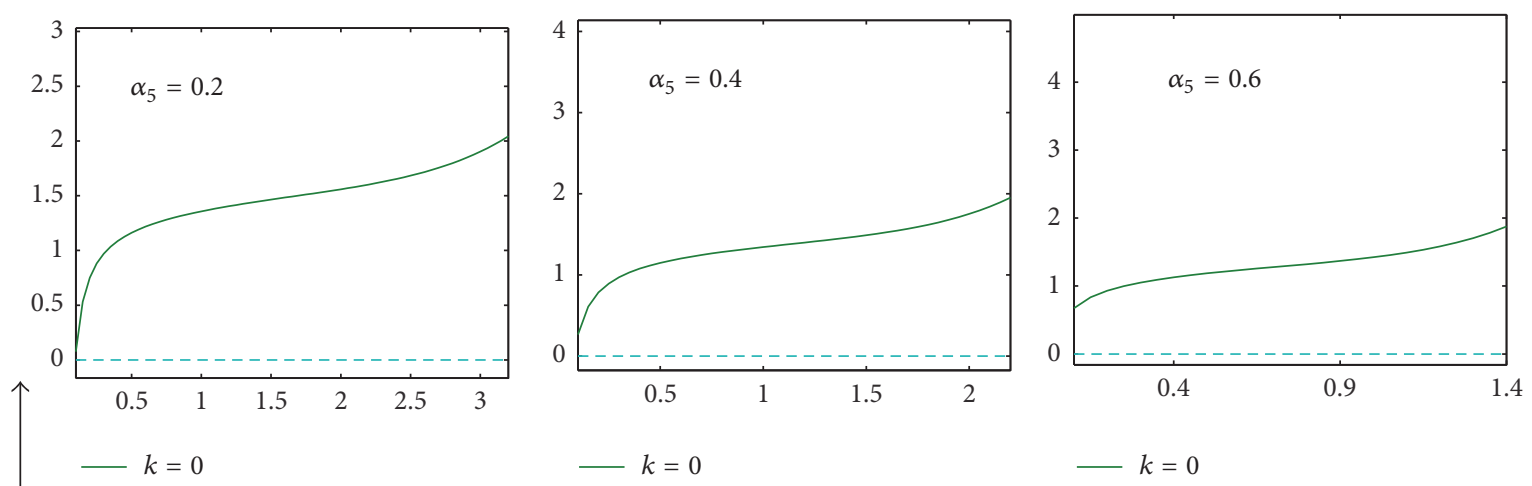

$-k=0$

$-k=0$
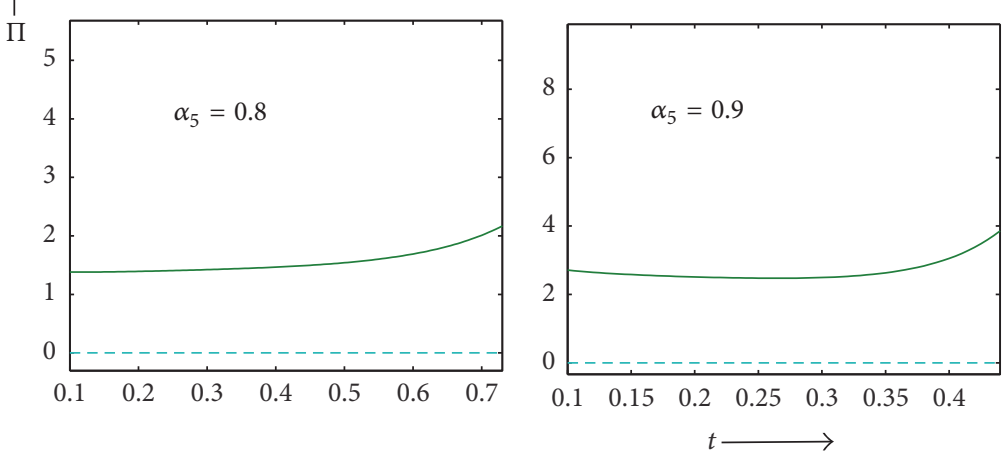

$-k=0$

$-k=0$

(b)

FIGURE 23: Variation of bulk viscous stress $\Pi$ against time for $\alpha_{1}=0.5, A=1, B=1, n=0.1, \omega=1, \phi_{0}=1, c_{2}=0.1$, and different $\alpha_{5}$. (b) shows the zooming of (a). 

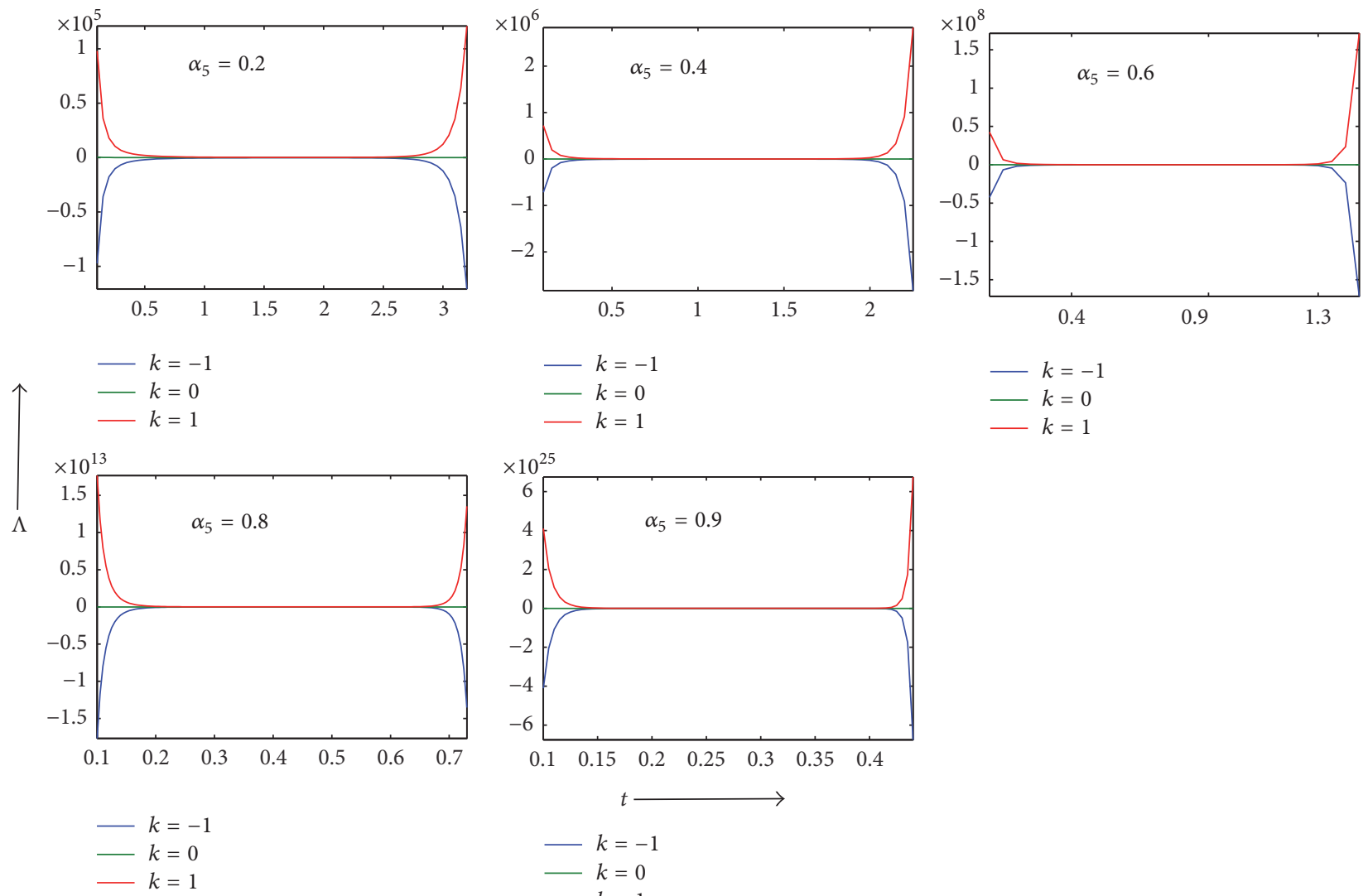
$-k=-1$
$k=0$
$-k=1$
$-k=-1$
$k=0$
$k=1$

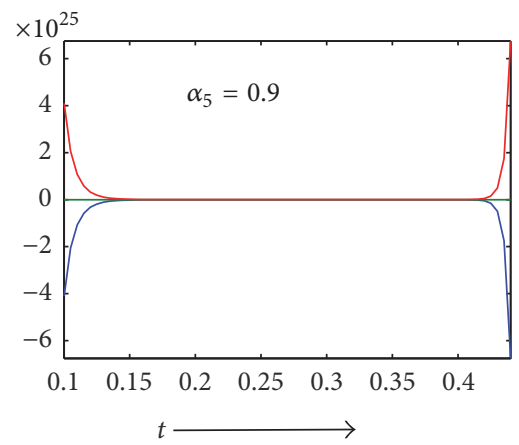

$$
\begin{aligned}
k & =-1 \\
-k & =0 \\
k & =1
\end{aligned}
$$

(a)
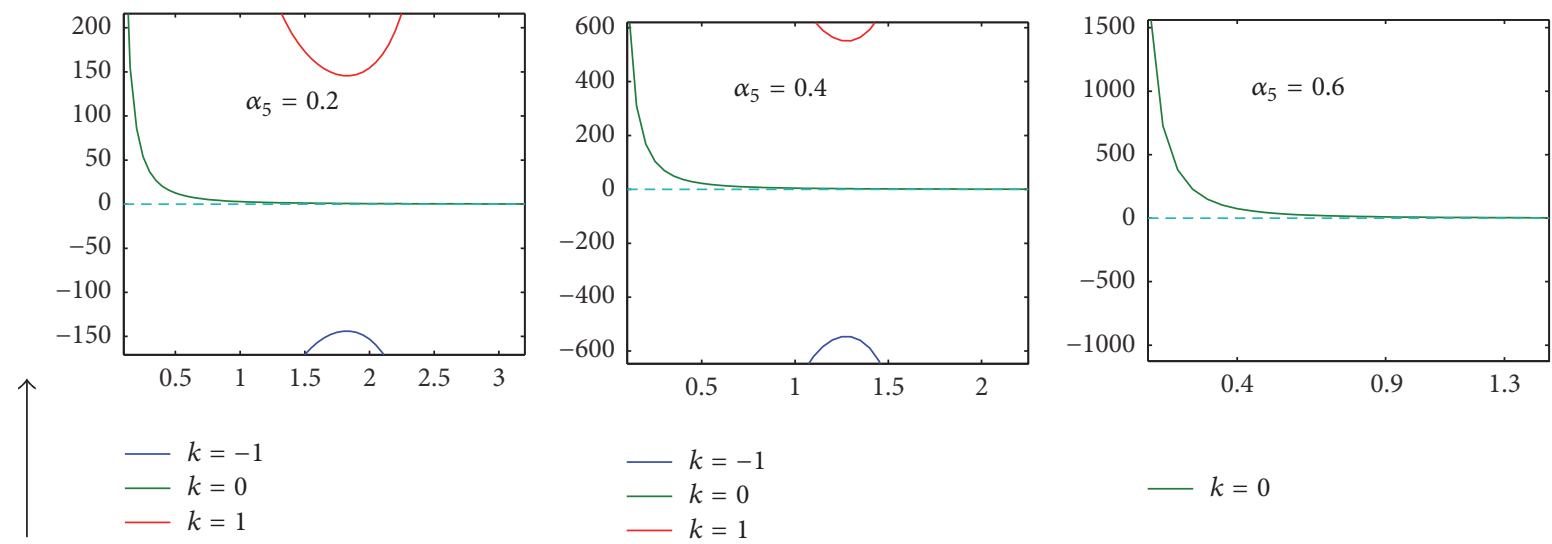

$$
\begin{aligned}
k & =-1 \\
k & =0
\end{aligned}
$$

$\Lambda$

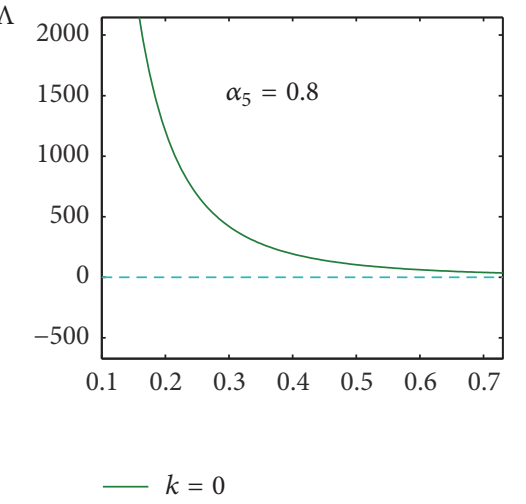

$-k=-1$

$-k=0$

$-k=1$

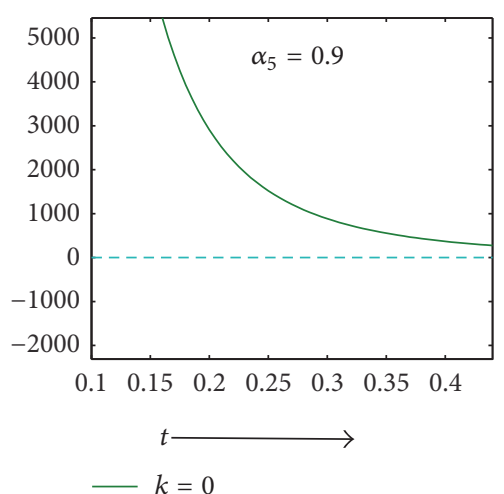

$-k=0$

(b)

FIGURE 24: Variation of cosmological constant $\Lambda$ against time for $\alpha_{1}=0.5, \omega=1, c_{2}=0.1$, and different $\alpha_{5}$. (b) shows the zooming of (a). 


\section{References}

[1] C. Brans and R. H. Dicke, "Mach's principle and a relativistic theory of gravitation," Physical Review, vol. 124, no. 3, pp. 925935, 1961.

[2] C. Mathiazhagan and V. B. Johri, "An inflationary universe in Brans-Dicke theory: a hopeful sign of theoretical estimation of the gravitational constant," Classical and Quantum Gravity, vol. 1, no. 2, pp. L29-L32, 1984.

[3] D. La and P. J. Steinhardt, "Extended inflationary cosmology," Physical Review Letters, vol. 62, no. 4, pp. 376-378, 1989.

[4] P. J. Steinhardt and F. S. Accetta, "Hyperextended inflation," Physical Review Letters, vol. 64, no. 23, pp. 2740-2743, 1990.

[5] C. Romero and A. Barros, "Does the Brans-Dicke theory of gravity go over to general relativity when $\omega \leftarrow \infty$ ?" Physics Letters A, vol. 173, no. 3, pp. 243-246, 1993.

[6] C. M. Will, Theory and Experiment in Gravitational Physics, Cambridge University Press, Cambridge, UK, 1981.

[7] V. Faraoni, Cosmology in Scalar-Tensor Gravity, Springer Science \& Business Media, 2004.

[8] V. B. Johri and K. Desikan, "Cosmological models with constant deceleration parameter in Brans-Dicke theory," General Relativity and Gravitation, vol. 26, no. 12, pp. 1217-1232, 1994.

[9] J. D. Barrow and J. Magueijo, "Solving the flatness and quasiflatness problems in Brans-Dicke cosmologies with a varying light speed," Classical and Quantum Gravity, vol. 16, no. 4, pp. 1435-1454, 1999.

[10] G. P. Singh and A. Beesham, "Bulk viscosity and particle creation in Brans-Dicke theory," Australian Journal of Physics, vol. 52, no. 6, pp. 1039-1049, 1999.

[11] A. A. Sen and N. Banerjee, "Nonstatic global string in BransDicke theory," Modern Physics Letters A. Particles and Fields, Gravitation, Cosmology, Nuclear Physics, vol. 15, no. 22-23, pp. 1409-1417, 2000.

[12] A. Barros and C. Romero, "Topological defects and gravitational forces in Brans-Dicke theory," Modern Physics Letters A. Particles and Fields, Gravitation, Cosmology, Nuclear Physics, vol. 16, no. 20, pp. 1297-1301, 2001.

[13] S. Chakraborty and A. Ghosh, "Inflationary scenario in BransDicke theory for Bianchi VIo space-time model," International Journal of Modern Physics D, vol. 12, no. 1, pp. 129-143, 2003.

[14] D. R. K. Reddy and V. Vijaya Lakshmi, "Bianchi type-V bulk viscous string cosmological model in scale-covariant theory of gravitation," Astrophysics and Space Science, vol. 353, no. 1, pp. 271-274, 2014.

[15] M. F. Shamir and A. A. Bhatti, "Anisotropic dark energy Bianchi type III cosmological models in the Brans-Dicke theory of gravity," Canadian Journal of Physics, vol. 90, no. 2, pp. 193-198, 2012.

[16] R. A. Knop, G. Aldering, R. Amanullah et al., "New constraints on $\Omega_{M}, \Omega_{\Lambda}$, and w from an independent set of 11 high-redshift supernovae observed with the Hubble Space Telescope," The Astrophysical Journal, vol. 598, no. 1, 102 pages, 2003.

[17] C. L. Bennet, R. S. Hill, G. Hinshaw, and M. L. Nolta, "Results from the COBE mission," Astrophysical Journal Supplement, vol. 148, p. 97, 2003.

[18] R. G. Vishwakarma, "A Machian model of dark energy," Classical and Quantum Gravity, vol. 19, no. 18, pp. 4747-4752, 2002.

[19] T. Singh and T. Singh, "Perfect fluid models of Bianchi type I in modified Brans-Dicke cosmology," Journal of Mathematical Physics, vol. 25, no. 9, pp. 2800-2802, 1984.
[20] L. O. Pimentel, "Exact cosmological solutions in the scalartensor theory with cosmological constant," Astrophysics and Space Science, vol. 112, no. 1, pp. 175-183, 1985.

[21] E. Ahmadi-Azar and N. Riazi, "A class of cosmological solutions of Brans-Dicke theory with cosmological constant," Astrophysics and Space Science, vol. 226, no. 1, pp. 1-5, 1995.

[22] T. Etoh, M. Hashimoto, K. Arai, and S. Fujimoto, "Age of the universe constrained from the primordial nucleosynthesis in the Brans-Dicke theory with a varying cosmological term," Astronomy and Astrophysics, vol. 325, no. 3, pp. 893-897, 1997.

[23] A. K. Azad and J. N. Islam, "Cosmological constant in the Bianchi type-I-modified Brans-Dicke cosmology," Pramana, vol. 60, no. 1, pp. 21-27, 2003.

[24] L.-E. Qiang, Y. Ma, M. Han, and D. Yu, "Five-dimensional Brans-Dicke theory and cosmic acceleration," Physical Review D - Particles, Fields, Gravitation and Cosmology, vol. 71, no. 6, article 061501, pp. 1-5, 2005.

[25] M. N. Smolyakov, "A small cosmological constant from the modified Brans-Dicke theory - an interplay between different energy scales," https://arxiv.org/abs/0711.3811.

[26] L. M. Reyes and J. E. M. Aguilar, "Embedding General Relativity with varying cosmological constant term in five-dimensional Brans-Dicke theory of gravity in vacuum," https://arxiv .org/abs/0902.4736.

[27] G. P. Singh, A. Y. Kale, and J. Tripathi, "Dynamic cosmological 'constant' in brans dicke theory," Romanian Journal of Physics, vol. 58, no. 1-2, pp. 23-35, 2013.

[28] H. Saadat and B. Pourhassan, "FRW bulk viscous cosmology with modified cosmic Chaplygin gas," Astrophysics and Space Science, vol. 344, no. 1, pp. 237-241, 2013.

[29] R. K. Mishra and A. Chand, "Cosmological models in alternative theory of gravity with bilinear deceleration parameter," Astrophysics and Space Science. An International Journal of Astronomy, Astrophysics and Space Science, vol. 361, no. 8, Paper No. 259, 10 pages, 2016.

[30] Y. L. Bolotin, V. A. Cherkaskiy, O. A. Lemets, D. A. Yerokhin, and L. G. Zazunov, "Cosmology In Terms Of The Deceleration Parameter. Part I," https://arxiv.org/abs/1502.00811.

[31] U. Debnath, "Modified Chaplygin gas with variable G and $\Lambda$," Chinese Physics Letters, vol. 28, no. 11, article 119801, 2011.

[32] G. C. Samanta, "Universe Described by Variable Modified Chaplygin Gas with Statefinder Diagnostic in General Relativity," International Journal of Theoretical Physics, vol. 53, no. 6, pp. 1867-1878, 2014. 

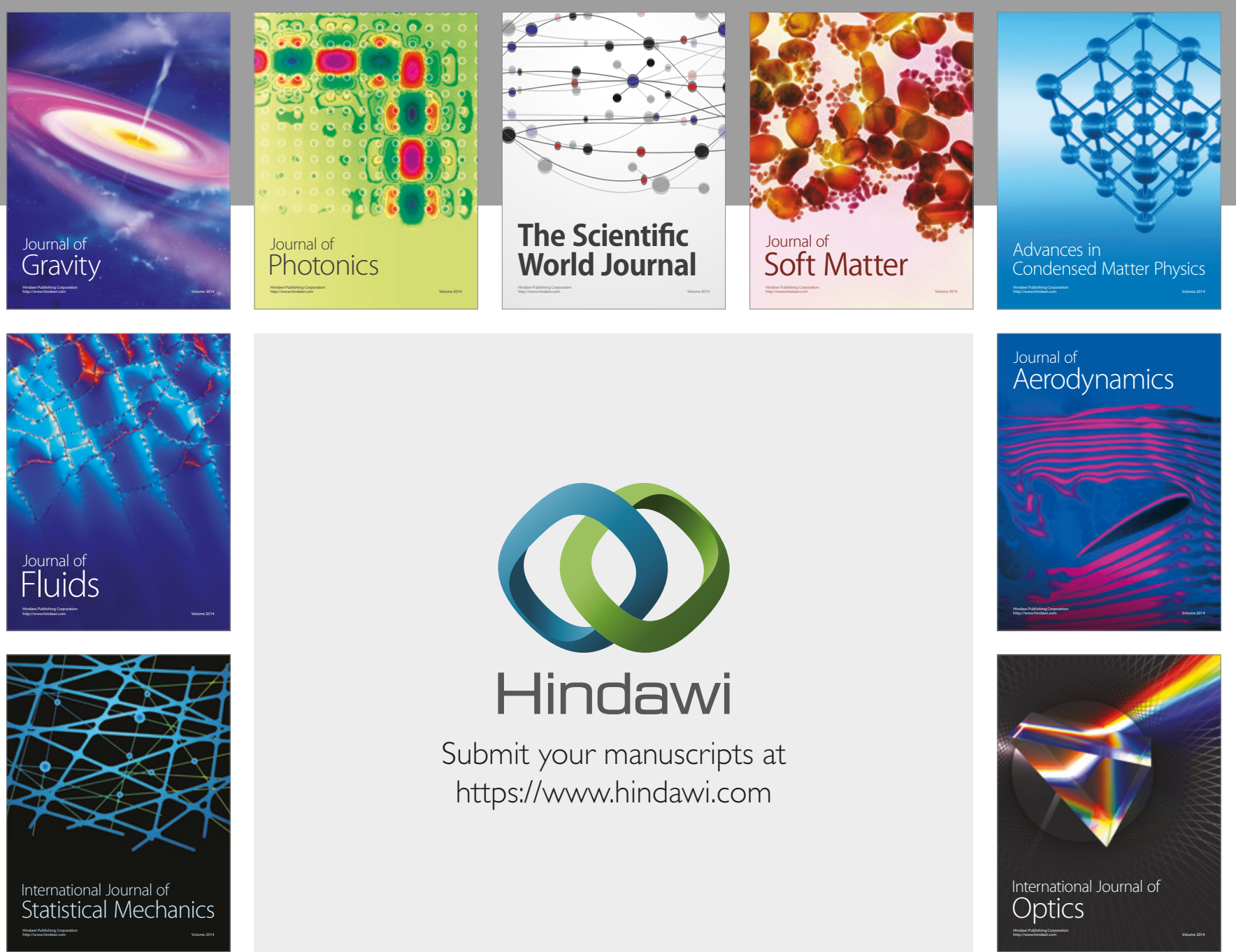

Submit your manuscripts at

https://www.hindawi.com
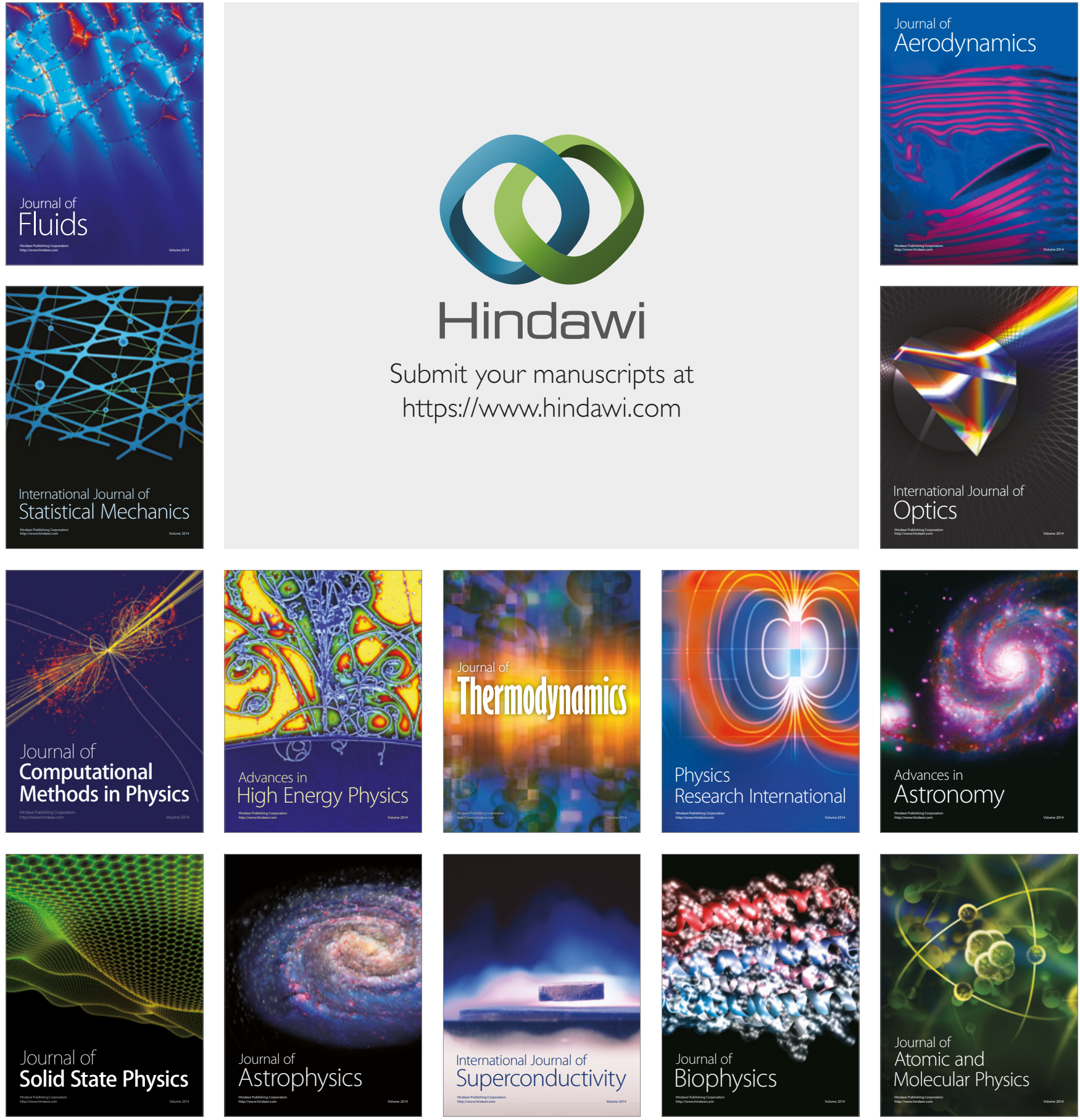